\title{
Guía clínica de calidad en colonoscopia y polipectomía
}

\author{
Omar Trujillo-Benavides ${ }^{*}$, Sergio Solana-Sentíes ${ }^{2}$, Jorge Aguilar-Mendoza ${ }^{3}$, Diego Angulo-Molina ${ }^{4}$, \\ Hugo Barrera-Torres 5 , Rafael Barreto-Zúñiga ${ }^{6}$, Reyes Betancourt-Linares ${ }^{7}$, Juan M. Blancas-Valencia ${ }^{8}$, \\ Jony Cerna-Cardona ${ }^{9}$, Héctor Espino-Cortés ${ }^{9}$, Angélica Hernández-Guerrero ${ }^{10}$, Daniel Keil-Ríos ${ }^{11}$, \\ Ma. Elena López-Acosta12, Clara L. Martínez-García ${ }^{13}$, Alejandra Noble-Lugo ${ }^{14}$, \\ Mario C. Peláez-Luna 15,16,17, Ángel A. Reyes-Dorantes ${ }^{18}$, Fernando Rojas-Mendoza ${ }^{19}$, \\ Xochiquetzal Sánchez-Chávez ${ }^{20}$, Sergio Solana-Sentíes², Omar E. Trujillo-Benavides ${ }^{1}$, \\ Felipe Zamarripa-Dorsey ${ }^{21}$ y Yolanda Zamorano-Orozco ${ }^{22}$
}

\begin{abstract}
${ }^{1}$ Servicio de Gastroenterología, Hospital General de Zona 42, Instituto Mexicano del Seguro Social, Puerto Vallarta, Jal.; ${ }^{2}$ Servicio de Endoscopia, Hospital Central Ignacio Morones Prieto, San Luis Potosí, S.L.P.; ${ }^{3}$ Servicio de Gastroenterología, Hospital Ángeles Querétaro, Querétaro, Qro.; ${ }^{4}$ Unidad de Endoscopia Avanzada, Centro Médico ABC, Ciudad de México; ${ }^{5}$ Centro Médico Puerta de Hierro, Zapopan, Jal.; ${ }^{6}$ Departamento de Endoscopia, Instituto Nacional de Ciencias Médicas y Nutrición Salvador Zubirán, Ciudad de México; ${ }^{7}$ Práctica privada, Chilpancingo, Gro.; ${ }^{8}$ Servicio de Endoscopia, Hospital de Especialidades Centro Médico Nacional Siglo XXI, Instituto Mexicano del Seguro Social, Ciudad de México; ${ }^{9}$ Servicio de Endoscopia, Hospital Juárez de México, Ciudad de México; ${ }^{10}$ Servicio de Endoscopia, Instituto Nacional de Cancerología, Ciudad de México; ${ }^{11}$ Departamento de Gastroenterología, Centro Médico ABC, Campus Observatorio, Ciudad de México; ${ }^{12}$ Servicio de Endoscopia, Hospital Ángeles Lomas, Estado de México; ${ }^{13}$ Servicio de Endoscopia, Hospital San Ángel Inn Universidad, Ciudad de México; ${ }^{14}$ Servicio de Gastroenterología, Hospital Español de México, Ciudad de México; ${ }^{15} \mathrm{Facultad}$ de Medicina, División de Investigación, Universidad Nacional Autónoma de México, Ciudad de México; ${ }^{16}$ Departamento de Gastroenterología, Instituto Nacional de Ciencias Médicas y Nutrición Salvador Zubirán, Ciudad de México; ${ }^{17}$ Unidad de Endoscopia Avanzada, Centro Médico ABC, Ciudad de México; ${ }^{18}$ Servicio de Gastroenterología, Hospital San Ángel Inn Chapultepec, Ciudad de México; ${ }^{19}$ Servicio de Gastroenterología y Endoscopia Digestiva, Fundación Clínica Médica Sur, Ciudad de México; ${ }^{20}$ Servicio de Endoscopia, Hospital Ángeles del Pedregal, Ciudad de México; ${ }^{21}$ Servicio de Gastroenterología, Hospital Juárez de México, Ciudad de México; ${ }^{22}$ Servicio de Endoscopia, Hospital General Regional N. ${ }^{\circ}$ Dr. Carlos MacGregor Sánchez-Navarro, Instituto Mexicano del Seguro Social, Ciudad de México. México
\end{abstract}

\section{Resumen}

La colonoscopia es el método de elección para el diagnóstico y tratamiento de la mayoría de las enfermedades de colon. Es la herramienta más eficaz en la prevención y detección oportuna de cáncer colorrectal (CCR), influyendo en su incidencia y mortalidad. Su eficacia y calidad depende de la habilidad del endoscopista, el porcentaje de área de mucosa examinada, el tiempo de examen y las complicaciones, entre otros. De tal forma que aunque existen indicadores aplicables a toda colonoscopia, existen otros específicos para cada indicación. La Asociación Mexicana de Gastroenterología (AMG) y la Asociación Mexicana de Endoscopia Gastrointestinal y Colegio de Profesionistas A. C. (AMEG) realizaron una reunión presencial en el mes de junio de 2019 a la que asistieron gastroenterólogos y endoscopistas de diversas instituciones del país para revisar y evaluar la evidencia actual sobre calidad en colonoscopia de escrutinio de CCR y desarrollar recomendaciones que favorezcan una colonoscopia de alta calidad.

Palabras clave: Calidad. Endoscopia. Colonoscopia. Guía clínica.

Correspondencia:

*Omar Trujillo-Benavides

E-mail: omaredel@ hotmail.com

E-mail: omaredenotmail.com
Fecha de recepción: 09-03-2021

Fecha de aceptación: 01-04-2021

DOI: 10.24875/END.21000012
Disponible en internet: 28-06-2021

Endoscopia. 2021;33(2):54-74

www.endoscopia-ameg.com 


\title{
Clinical guidelines in quality in colonoscopy and polypectomy
}

\begin{abstract}
Colonoscopy is the method of choice for diagnosis, and treatment of most colon diseases, being possibly the most effective tool in the prevention and timely detection of colorectal cancer (CRC), influencing its incidence and mortality. Efficacy and diagnostic and therapeutic quality depend on the ability of the endoscopist, percentage of colonic mucosa examined, the examination time, complications, among others. In such a way that even though there are indicators applicable to all colonoscopy, there are other specific ones for each indication. The Mexican Association of Gastroenterology (AMG) and the Mexican Association of Gastrointestinal Endoscopy and Professional College AC (AMEG) held a face-to-face meeting in June 2019, which was attended by gastroenterologists and endoscopists to review and evaluate the current evidence on the quality of CRC screening colonoscopy and develop recommendations that favor the performance of a high-quality colonoscopy.
\end{abstract}

Key words: Quality. Endoscopy. Colonoscopy. Clinical guideline.

\section{Introducción}

Una endoscopia de calidad es aquella que cumple con los objetivos para los que se realiza y/o indica. La calidad puede medirse comparando el desempeño de un individuo o un grupo de individuos respecto a un punto de referencia.

Existen indicadores de calidad, que son instrumentos de medición, basados en hechos y datos, que permiten evaluar la calidad de los procesos, productos y servicios para asegurar la satisfacción de los clientes, es decir, miden el nivel de cumplimiento de las especificaciones establecidas para una determinada actividad o proceso.

La colonoscopia es el método de elección para el diagnóstico, y en muchos casos para el tratamiento, de la mayoría de las enfermedades de colon, siendo posiblemente la herramienta más eficaz en la prevención y detección oportuna de cáncer colorrectal (CCR) e influyendo en su incidencia y mortalidad. Su calidad dependerá de si cumple con los objetivos establecidos de acuerdo con la indicación y desenlaces esperados y/o deseados.

La eficacia y calidad diagnóstica y terapéutica depende tanto de la habilidad del endoscopista como del porcentaje de área de mucosa colónica examinada, el tiempo de examen y las complicaciones, entre otros. De tal forma que aunque existen indicadores aplicables a toda colonoscopia, existen otros específicos para cada indicación. La calidad de una colonoscopia de escrutinio de CCR se medirá con indicadores distintos a los utilizados en una colonoscopia por hemorragia (p. ej., tasa de detección de adenomas [TDA], adecuada descripción y clasificación macroscópica, polipectomía completa, etc.).
Debido al papel central que tiene la colonoscopia en la detección oportuna de CCR y su impacto sobre la incidencia y mortalidad asociadas, se han desarrollado criterios que califican la calidad de toda colonoscopia de escrutinio.

La Asociación Mexicana de Gastroenterología (AMG) y la Asociación Mexicana de Endoscopia Gastrointestinal y Colegio de Profesionistas A. C. (AMEG) realizaron una reunión presencial en el mes de junio de 2019 a la que asistieron gastroenterólogos y endoscopistas de diversas instituciones públicas y privadas del país para revisar y evaluar la evidencia actual sobre calidad en colonoscopia de escrutinio de CCR y desarrollar recomendaciones que favorezcan la realización de una colonoscopia de alta calidad.

\section{Metodología}

Para la elaboración de esta guía clínica se utilizó el método Delphi'. Se crearon cuatro mesas de trabajo y a cada una se le asignó un tema específico que representa cada una de las secciones de la presente guía. Tres de los participantes realizaron una búsqueda en la base de datos PubMed de artículos publicados a manera de resumen o artículo completo en los idiomas inglés y español. Se dio preferencia a revisiones sistemáticas, metaanálisis, guías de práctica clínica, consensos, ensayos clínicos controlados y cohortes, aunque no se limitó a este tipo de manuscritos.

Una vez concluida la búsqueda se elaboraron enunciados relacionados con el tema de cada mesa, mismos que fueron evaluados y votados por el comité organizador y fueron enviados a cada coordinador de mesa junto con la bibliografía seleccionada. Cada coordinador asignó aleatoriamente uno o más 
enunciados a cada participante, quien calificó la evidencia que los apoya. Para calificar la evidencia se utilizó el sistema GRADE (Grading of Recommendations, Assessment, Development and Evaluation) modificado para calificar la calidad de la evidencia publicada y seleccionar los artículos más relevantes sin importar si los resultados eran positivos 0 negativos ${ }^{2}$.

Con la finalidad de homogeneizar la forma de evaluación y que cada participante conociera el método, se les pidió y envió un enlace electrónico para concluir el curso en línea gratuito UpToDate del sistema GRADE. Este sistema tiene el objetivo de mejorar la evaluación de la calidad de la evidencia y la fuerza con la que esta se recomienda. En este sistema la graduación de la calidad no se basa en el tipo de estudio (diseño y metodología), se consideran también los desenlaces (pertinencia, relevancia, etc.), así como las preguntas elaboradas para estudiar dicho desenlace.

De tal forma que aunque la evidencia de mayor calidad se origina en revisiones sistemáticas, ensayos clínicos, etc., el sistema GRADE clasifica la calidad con base en el diseño y los métodos utilizados para evaluar los desenlaces preseleccionados o responder una pregunta tipo PICO (Población o Pacientes, Intervención o Indicador, Control o Comparación y $\mathrm{O}$ de desenlace, por su traducción del inglés Outcome) elaborada con anterioridad y con fines específicos. Esto permite que evidencia de menor calidad (p. ej., casos y controles, estudios transversales, series de casos) pueda ser considerada ${ }^{3}$.

La calidad de la evidencia puede ser «alta» cuando la seguridad o confianza en los desenlaces o efectos estimados no puede o no podría ser modificada por investigaciones subsecuentes. Se considera «moderada" cuando investigaciones posteriores podrían modificar los efectos o desenlaces y, por lo tanto, nuestra confianza en la información actual disminuye. La calidad es "baja» cuando es muy probable que la información actual se modifique con estudios ulteriores y «muy baja» cuando los desenlaces o efectos en estudio conocidos en la actualidad son extremadamente inciertos.

La calidad y fuerza de recomendación (débil o fuerte) se expresan como letras mayúsculas de la $A$ la $D$ (que indican la calidad de evidencia, A para muy alta calidad y $\mathrm{D}$ para muy baja calidad) y números (que indican la fuerza de la recomendación a favor 0 en contra, 1 fuerte y 2 débil) (Tabla Recomendaciones GRADE). Estos enunciados y su calificación fueron presentados por los coordinadores a los miembros de sus mesas, donde se revisó su contenido y redacción, efectuando modificaciones en conjunto, además de discutir la pertinencia o necesidad de eliminar o agregar otros enunciados. Se votó «en acuerdo» o «en desacuerdo» para cada enunciado. Cuando el acuerdo era mayor al $75 \%$ se concluyó que el enunciado permanecería sin modificaciones, y cuando el acuerdo era menor al $75 \%$ se realizaron modificaciones en contenido y redacción sometiéndose nuevamente a votación, y así sucesivamente hasta llegar a un acuerdo mayor al $75 \%$.

Aquellos enunciados con desacuerdo mayor al $75 \%$, repetidos o redundantes, fueron eliminados del proceso. Posterior a la reunión cada coordinador y miembros de cada mesa desarrollaron comentarios y consideraciones finales a cada enunciado, además de revisar y determinar el nivel y calidad de evidencia que lo apoya, así como la fortaleza de la recomendación, basados en el sistema modificado GRADE antes mencionado (Tabla Síntesis de las recomendaciones).

Los indicadores de calidad en una colonoscopia incluyen indicadores previos al procedimiento (consentimiento informado, valoración por anestesiología, identificación de comorbilidades y factores de riesgo manejo de antiagregantes y anticoagulantes, condiciones que requieran de antibióticos profilácticos, etc.), indicadores durante el procedimiento (limpieza, intubación cecal, TDA, polipectomía completa, tiempo de retirada, complicaciones inmediatas) e indicadores posprocedimiento (recuperación, molestias y complicaciones tardías asociadas al procedimiento, evaluación prealta y seguimiento). La presente guía abordará principalmente los indicadores relacionados con el procedimiento.

Esta guía puede y deberá ser revisada y editada periódicamente de acuerdo con la necesidad de incorporar cambios y avances tecnológicos, así como información nueva y relevante. Las recomendaciones presentadas y su fuerza se basan en la revisión de la evidencia científica disponible al momento de su elaboración, así como en la discusión y evaluación de los riesgos y beneficios hecha por los participantes de la guía. Es importante recordar que esta, como todas las guías, presenta información que pretende ayudar a los involucrados en el cuidado de pacientes y no deben considerarse reglas ni estándares de cuidado inmodificables, puesto que las decisiones clínicas deben individualizarse y suelen ser resultado de un análisis detallado de la situación clínica del paciente y recursos disponibles que pudieran estar o no estar en acuerdo con este documento. 


\section{Preparación para la colonoscopia}

\section{Preparación}

\section{EL GRADO DE LIMPIEZA INTESTINAL ES UN INDICADOR DE CALIDAD EN COLONOSCOPIA}

Recomendación fuerte. Nivel de evidencia alto (GRADE 1A)

La colonoscopia es el estudio de elección para visualizar la mucosa del colon y evaluación de síntomas y patología colónica, además de que tiene un papel destacado en el escrutinio de $\mathrm{CCR}^{4}$.

La eficacia diagnóstica y terapéutica de la colonoscopia depende de la calidad de la preparación. Una limpieza colónica inadecuada disminuye la detección de lesiones e incrementar el riesgo de eventos adversos asociados al procedimiento ${ }^{5}$. Hasta el $20-25 \%$ de las colonoscopias no cuentan con una adecuada preparación ${ }^{6-8}$. Bowles, et al. realizaron un estudio prospectivo de 9,223 colonoscopias en el cual encontraron que una de cada cinco colonoscopias fallidas se relacionó con una mala preparación ${ }^{9}$.

Nelson, et al. realizaron un estudio multicéntrico que analizó los resultados de 3,196 colonoscopias realizadas a pacientes entre 50 y 75 años. Los pacientes con preparación inadecuada tuvieron una tasa de colonoscopia incompleta significativamente mayor comparada con aquellos con una preparación adecuada (19.3 vs. $2.2 \% ; p=0.001)^{10}$.

La limpieza adecuada del colon también se relaciona con un menor tiempo de estudio, pues reduce tanto el tiempo de intubación cecal como el tiempo de retirada del colonoscopio. Froelich, et al. demostraron que el tiempo de retirada del colonoscopio fue significativamente menor en pacientes con preparación adecuada en comparación con aquellos con preparación inadecuada (9.8 vs. $11.3 \mathrm{~min} ; \mathrm{p}<0.001)^{6}$.

\section{SE RECOMIENDA INCLUIR EL GRADO DE LIMPIEZA DEL} COLON EN EL INFORME DE COLONOSCOPIA, UTILIZANDO LA ESCALA DE PREPARACIÓN INTESTINAL DE BOSTON

Recomendación fuerte. Nivel de evidencia moderado (GRADE 1B)

Es importante documentar la calidad de la limpieza intestinal lograda. Una preparación adecuada se define como aquella que permite la detección de lesiones $\leq 5 \mathrm{~mm}$ de manera confiable ${ }^{11}$.

La calificación de la calidad de la preparación colónica expresarse de manera objetiva, utilizando escalas validadas, como la escala Aronchick, la puntuación de preparación intestinal de Boston (BBPS), la escala de Ottawa, la escala de limpieza de Harefield y la de Chicago $^{11-13}$.

Las puntuaciones de Ottawa y BBPS requieren que el endoscopista obtenga una valor numérico del estado de limpieza intestinal en cada segmento del colon. El resultado de la adición de cada uno de estos valores indica el grado de «limpieza intestinal global». Este puntaje permite calificar la preparación como excelente, buena, suficiente o inadecuada.

\section{Escala de Boston}

La BBPS es quizá la escala más conocida y utilizada. El puntaje debe asignarse una vez que se ha realizado la aspiración de los residuos contenidos en cada segmento.

De acuerdo con la cantidad de mucosa observada y cantidad y características de los residuos no aspirados, en cada segmento (colon derecho, colon transverso y colon izquierdo) se asigna un puntaje de 0 a 3 :

- 0 corresponde a un segmento no preparado y mucosa no visible por la presencia de heces solidas que no removibles.

- 1 indica que se observan porciones de mucosa, pero otras no debido a manchas, residuos fecales o residuos opacos líquidos.

- 2 existe escasa cantidad de manchas o residuos fecales u opacos líquidos, pero se observa bien la mucosa del segmento.

- 3 se observa la totalidad de la mucosa del segmento, sin manchas, residuos fecales u opacos líquidos.

Los puntajes individuales de cada segmento se suman y se obtiene un resultado global (0-9 puntos). Un puntaje mayor indica mejor preparación (Fig. 1) ${ }^{13}$.

Existe una asociación directamente proporcional entre el puntaje de la BBPS y la TDA ${ }^{13,14}$. Kim, et al. reportaron que la BBPS tiene una concordancia interobservador fuerte, con coeficiente de correlación interclase (ICC) de 0.90 con valor kappa de 0.63 (intervalo de confianza del 95\% [IC 95\%]: 0.57-0.68) en los distintos rangos de puntaje ${ }^{14}$.

En una revisión sistemática, la BBPS obtuvo una excelente concordancia interobservador (ICC, IC 95\%: 0.74-0.91) y se asoció con mayor detección de pólipos en colon izquierdo (razón de momios [RM]: 2.58), colon derecho (RM: 1.6) y a menor necesidad de repetir la colonoscopia (en puntaje global $\geq 5$ ). También se asoció a un menor tiempo de inserción y retirada $(p<0.001)^{15,16}$. 


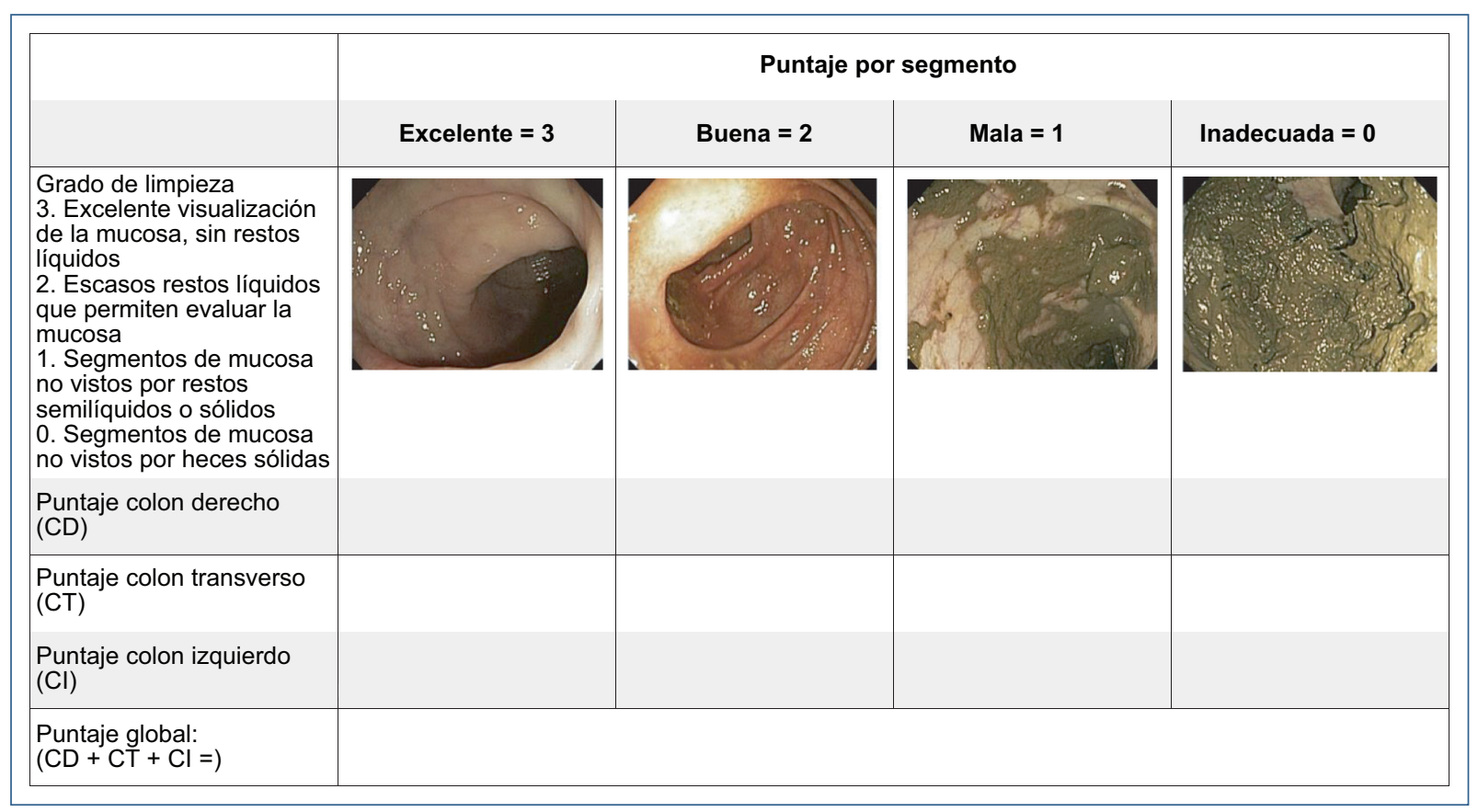

Figura 1. Escala de preparación intestinal de Boston (adaptada de Lorenzo-Zúñiga, et al., 20126).

Incluso cuando la elección de la escala para calificar la calidad de preparación depende completamente de cada endoscopista, con el objetivo de homogeneizar los criterios de evaluación sugerimos se utilice la BBPS e integrarla al reporte final.

\section{CuANDO LA PREPARACIÓN DE COLON ES INADECUADA DURANTE COLONOSCOPIAS DE ESCRUTINIO DE CCR EN POBLACIÓN CON RIESGO PROMEDIO O DE SEGUIMIENTO DE PÓLIPOS, SE RECOMIENDA REPETIR LA COLONOSCOPIA EN EL TRANSCURSO DE UN AÑO}

Recomendación fuerte. Nivel de evidencia moderado (GRADE 1B)

Existen factores independientes que afectan a la preparación de los pacientes (p. ej., realización del estudio al final del día, pacientes hospitalizados, uso de antidepresivos tricíclicos e incapacidad para seguir las instrucciones de la preparación, entre otros), que deben considerarse al momento de indicar la preparación del colon. Una preparación inadecuada del coIon aumenta la posibilidad de CCR de intervalo. En un estudio retrospectivo en el que solo el $31.9 \%$ de las colonoscopias de escrutinio para CCR tuvieron una preparación aceptable se encontraron adenomas en el $28 \%$ de las colonoscopias realizadas tres años después, de los cuales el $2.7 \%$ fueron adenomas avanzados ${ }^{17}$. En un estudio retrospectivo de pacientes en quienes se repitió una colonoscopia un año después, se reportó una tasa de adenomas no detectados hasta del $35 \%{ }^{8}$.

Cuando no se logra una preparación adecuada la colonoscopia deberá reprogramarse. No existe un consenso sobre el tiempo adecuado para repetir una colonoscopia en un paciente con preparación inadecuada.

Lebowhl, et al. instruyeron a los pacientes con mala preparación sobre cómo mejorarla, con el fin de realizar un estudio de «rescate» al día siguiente, pero solo el $47 \%$ de los pacientes reprogramados siguieron las indicaciones?.

El puntaje de la BBPS puede determinar el tiempo para repetir la colonoscopia de seguimiento. En pacientes con colonoscopia negativa, con puntaje global de la BBPS $\geq 6$ y/o $\geq 2$ por segmento en los tres segmentos, el seguimiento puede hacerse a los 10 años; si el puntaje global es $<60<2$ por segmento, el procedimiento debe repetirse en el transcurso de un año ${ }^{18}$.

Nuestra recomendación en los pacientes con una preparación inadecuada es que se repita la colonoscopia con una preparación más intensa en un lapso no mayor de un año. 
LA EDUCACIÓN AL PACIENTE SOBRE LA PREPARACIÓN INTESTINAL MEJORA EL APEGO A LAS INDICACIONES Y LA CALIDAD DE LA PREPARACIÓN

Recomendación fuerte. Nivel de evidencia alto (GRADE 1A)

El desconocimiento de la importancia de un preparación y limpieza intestinal adecuadas explica la pobre adherencia a las instrucciones, e incrementa la tasa de preparación intestinal inadecuada.

El equipo de salud debe proporcionar información correcta y clara que mejore el apego del paciente. Se debe enfatizar la importancia del cumplimiento de las indicaciones y el impacto de una adecuada limpieza intestinal en los hallazgos y resultados de la colonoscopia.

Se han diseñado diversas intervenciones encaminadas a educar al paciente sobre la calidad de su preparación intestinal previa al estudio. Para esto se han evaluado medios verbales, escritos, visuales, medios electrónicos y redes sociales (aplicaciones de celular, mensajes, SMS), entre otros ${ }^{5,11}$.

Un par de metaanálisis sugieren que las intervenciones educativas previas a la colonoscopia, tanto en pacientes ambulatorios como en hospitalizados, mejoran significativamente la calidad de preparación intestinal (risk ratio [RR]: 1.22; IC 95\%: 1.10-1.36) e incrementan el número de pacientes con adecuada preparación (RM: 3.49; IC 95\%: 1.67-7.28; $p=0.0009$ ), aunque no siempre incrementan la tasa de detección de pólipos (TDP) (RR: 1.14; IC 95\%: 0.87-1.51) o en la necesidad de repetir la colonoscopia (RR: 0.52; IC 95\%: $1.25-1.04)^{19,20}$.

Park, et al. ${ }^{21}$, utilizando el puntaje global de Ottawa, demostraron que el uso de un vídeo educativo incrementa el número de pacientes con una preparación excelente ( $3.03 \pm 1.9$ vs. $4.21 \pm 1.9 ; p<0.001)$ o buena (< 6: 91.6 vs. $78.5 \%$; $p<0.001$ ), en comparación con los que no recibieron instrucción adicional por medio de un vídeo, respectivamente.

La instrucción personalizada comparada con una convencional incrementa el porcentaje de pacientes con una preparación adecuada. (62\%; IC 95\%: 53-70\% vs. $35 \%$; IC 95\%: $26-44 \%$; $p<0.001$ respectivamente). El efecto de la personalización logra un mayor impacto en pacientes menores de 65 años (67 vs. $35 \%$; $p<0.001$ ), hombres (60 vs. $33 \% ; p=0.003$ ), aquellos con niveles educativos más altos (68 vs. $37 \%$; $p=0.002$ ), en aquellos que viven en zonas urbanas (68 vs. $40 \% ; p=0.004$ ) y en aquellos con colonoscopia previa (68 vs. $40 \% ; p=0.001)^{22}$.
El uso de estrategias especiales como folletos con caricaturas, Ilamadas a celular, SMS, aplicaciones de celulares y redes sociales resulta en una mejor calidad de preparación intestinal que aquellos que reciben instrucciones convencionales (RM: 2.35; IC 95\%: 1.653,$35 ; p \leq 0.001$ ). Adicionalmente, los pacientes que reciben instrucciones mediante estrategias especiales muestran mayor disposición a repetir la preparación (RM: 1.91; IC 95\%: 1.20-3.04; $p=0.006)^{23}$.

El proporcionar instrucciones adecuadas al paciente sobre la relevancia clínica de la calidad de la preparación previo al estudio impacta favorablemente en la calidad de la preparación intestinal y es tan importantes como el método de preparación.

Recomendamos que los pacientes que serán sometidos a colonoscopia reciban información clara, precisa y completa que favorezca su apego a las indicaciones de preparación.

\section{LA CALIDAD DE LA PREPARACIÓN EN PACIENTES}

HOSPITALIZADOS MEJORA AL PROPORCIONAR

EDUCACIÓN AL PERSONAL DE ENFERMERÍA ACERCA DE CÓMO REALIZAR UNA ADECUADA SUPERVISIÓN DEL PROCESO DE LIMPIEZA INTESTINAL

Recomendación fuerte. Nivel de evidencia bajo (GRADE 1C)

La educación al personal de enfermería sobre los procedimientos de preparación intestinal y evaluación de la calidad de estos previo a una colonoscopia es un área de oportunidad. Se ha reportado que tan solo un $34 \%$ conoce las indicaciones dietéticas, entre el 39 y el $51 \%$ conocen los horarios adecuados para la administración de las diferentes dosis en esquemas de la preparación de dosis dividida. Solo el $60 \%$ de las enfermeras pueden identificar una preparación adecuada conforme a la apariencia de las evacuaciones ${ }^{24}$.

La falta de capacitación del personal de enfermería es un factor de riesgo independiente para una preparación inadecuada (RM: 2.365; $p=0.025)$. La capacitación del personal de enfermería respecto a la manera de llevar a cabo y supervisar la preparación para colonoscopia en pacientes hospitalizados mejora el cumplimiento de la dieta e indicaciones, así como la calidad de preparación (puntaje global de Ottawa: $4.42 \pm 2.23$ vs. $6.15 \pm 2.38 ; p<0.001$ ) y número de pacientes con preparación adecuada (58.8 vs.31.1\%; $p<0.001$ ). Adicionalmente, disminuye la ansiedad y mejora la satisfacción de los pacientes ${ }^{25}$. 


\section{ES INDISPENSABLE REALIZAR UNA DIETA BAJA EN RESIDUO DURANTE LA PREPARACIÓN PREVIA A LA COLONOSCOPIA}

Recomendación fuerte. Nivel de evidencia moderado (GRADE 1B)

La dieta se considera un elemento fundamental para la preparación previa a una colonoscopia. Tradicionalmente recomienda una dieta líquida o sin residuo. Ambas opciones son efectivas y seguras, sin que existan diferencias en la calidad de la preparación (RM: 1.21; IC 95\%: 0.64-2.28; $p<0.58$ ) ni en los efectos adversos (RM: 0.88; IC 95\%: 0.58-1.35; $p<0.57$ ), aunque la dieta baja en residuo es más aceptada (RR: 1.06; IC 95\%: 1.02-1.11) y los pacientes en esta dieta tienen mayor disponibilidad en repetir tanto la dieta como la preparación para colonoscopia (RM: 1.86; IC 95\%: 1.34-2.59; $\mathrm{p}<0.01)^{26,27}$.

El tiempo previo al estudio que debe seguirse la dieta no es relevante; un estudio que comparó la efectividad de la dieta sin residuo por 3 días vs. 1 día, utilizando la BBPS no encontró diferencias significativas con relación al apego a la dieta, satisfacción del paciente en la TDP y TDA ${ }^{28,29}$.

La dieta líquida o baja en residuo son comparables en sus resultados sobre la calidad de preparación intestinal y su elección dependerá de las preferencias del médico y paciente (Tabla 1).

\section{EL MÉTOdO DE PREPARACIÓN UTILIZANDO 4 LITROS DE POLIETILENGLICOL EN DOSIS DIVIDIDA ES SEGURO Y EFECTIVO}

Recomendación fuerte. Nivel de evidencia alto (GRADE 1A)

\section{Tipos de preparación para la colonoscopia}

La preparación ideal para colonoscopia debe permitir una limpieza óptima del colon, sin producir desequilibrio hidroelectrolítico en el paciente y ser segura incluso en pacientes con comorbilidades. Debe ser en lo posible bien tolerada por el paciente, sin causar incomodidad y de un costo accesible. De acuerdo con su osmolaridad, las preparaciones pueden ser clasificadas en: isoosmolares (p. ej., polietilenglicol), hiperosmolares (p. ej., fosfato de sodio [NaP]) o hipoosmolares ${ }^{30}$.

Los agentes isoosmolares son osmóticamente balanceados y no fermentables, por lo que producen mínimos cambios hidroelectrolíticos. Los agentes hiperosmolares producen un flujo significativo de agua y electrolitos hacia el lumen intestinal que resulta en mayor riesgo de deshidratación y alteraciones electrolíticas ${ }^{31}$.

La preparación más conocida en el país es aquella que utiliza PEG disuelto en 4 litros de agua (4L PEG). Esta solución debe ser ingerida por el paciente en el transcurso de 4 horas, limitando significativamente el apego y tolerancia. Es por esto que se han buscado alternativas con bajo volumen que ofrezcan una eficacia y un perfil de seguridad semejantes.

La solución de 4L PEG administrada en dosis dividida en comparación con otros métodos de preparación (4L PEG monodosis, uso de bisacodilo, preparaciones de bajo volumen) logra una limpieza buena o excelente en una proporción significativamente mayor de pacientes (RM: 3.4; IC 95\%: 2.45-4.89; $p=0.008$ ), sin que existan diferencias significativas en satisfacción, cumplimiento ni disposición para repetir la preparación a futuro por parte de los pacientes, así como tampoco en eventos adversos ${ }^{32}$.

\section{LA PREPARACIÓN DE BAJO VOLUMEN DE} POLIETILENGLICOL CON BISACODILO Y EL PICOSULFATO DE SODIO CON CITRATO DE MAGNESIO SON ALTERNATIVAS CON ADECUADA EFECTIVIDAD

Recomendación fuerte. Nivel de evidencia alto (GRADE 1A)

La preparación con 2 litros de PEG (2L PEG) en combinación con bisacodilo (BIS), así como la preparación a base de picosulfato de sodio con citrato de magnesio (PSCM), son alternativas de bajo volumen que han sido ampliamente utilizadas.

- PEG de bajo volumen + BIS. En México se dispone de una preparación isoosmolar de bajo volumen a base de 2L PEG combinado con BIS (2L PEG + BIS). Comparada $^{33-35}$ con la preparación de $4 \mathrm{~L}$ PEG, la preparación 2L PEG + BIS logró con mayor frecuencia puntajes de preparación excelente (RM: 1.24), sin diferencias en la frecuencia de preparación satisfactoria o inadecuada; tampoco se encontraron diferencias en la TDA (RM: 0.85). Sin embargo, la preparación con 2L PEG + BIS se asocia a menos eventos adversos, como náuseas (RM: 0.57), vómitos (RM: 0.57) y distensión abdominal (RM: 0.65) y un mayor número de pacientes en este grupo, están dispuestos a repetir el régimen en ocasiones futuras.

Aunque dosis altas de BIS pueden asociarse con colitis isquémica, esta no se reportó en los metaanálisis previos que incluyen estudios en los que se 
Tabla 1. Dieta baja en residuo

\begin{tabular}{|c|c|c|}
\hline Grupo de alimentos & Ejemplo de alimentos permitidos & Ejemplo de alimentos no permitidos \\
\hline Pan y almidones & $\begin{array}{l}\text { Pan blanco, pan tostado, bolillo, hot cakes, waffles. } \\
\text { Galletas saladas } \\
\text { Cereales fríos refinados hechos de arroz, maíz o avena. } \\
\text { Arroz blanco } \\
\text { Pasta refinada blanca } \\
\text { Papa cocida sin cáscara } \\
\text { Tortilla }\end{array}$ & $\begin{array}{l}\text { Pan integral, multigrano o con salvado } \\
\text { Galletas integrales } \\
\text { Cereales integrales o granola } \\
\text { Arroz integral o silvestre } \\
\text { Pasta integral, quinoa } \\
\text { Papa cocida con cáscara } \\
\text { Tortilla frita }\end{array}$ \\
\hline $\begin{array}{l}\text { Lácteos y } \\
\text { derivados }\end{array}$ & $\begin{array}{l}\text { Lácteos bajos en grasa: leche, queso suave, queso } \\
\text { cottage, requesón, yogur natural simple. Leche de } \\
\text { almendra, soya, o arroz. Flan }\end{array}$ & $\begin{array}{l}\text { Leche entera } \\
\text { Quesos secos o grasos } \\
\text { Helado }\end{array}$ \\
\hline Frutas y vegetales & $\begin{array}{l}\text { Caldo de verduras colado } \\
\text { Frutas cocidas, sin semilla ni cáscara, en conserva o } \\
\text { enlatadas } \\
\text { Frutas sin semilla y sin cáscara, plátano, melón, } \\
\text { papaya, durazno, sandía (sin semilla) } \\
\text { Aguacate, zanahoria cocida } \\
\text { Puré de manzana }\end{array}$ & $\begin{array}{l}\text { Vegetales crudos o parcialmente cocinados } \\
\text { Hortalizas y tubérculos } \\
\text { Frutos secos: piña, ciruela, higo, frutos del bosque } \\
\text { Jugos con pulpa. Salsa de tomate } \\
\text { Chícharos, alubias, habas, lentejas, frijol, garbanzo } \\
\text { Brócoli, apio, germen de soya }\end{array}$ \\
\hline Carnes y huevo & $\begin{array}{l}\text { Carne de res magra, molida o bien cocinada } \\
\text { Jamón, cerdo, pescado blanco, mariscos } \\
\text { Pollo (sin piel). Huevos cocidos }\end{array}$ & $\begin{array}{l}\text { Carne fibrosa o grasosa } \\
\text { Embutidos, cordero, pescado grasoso (azul o en } \\
\text { aceite) } \\
\text { Pollo con piel, huevos fritos }\end{array}$ \\
\hline Bebidas & Agua simple, café, te & Refrescos \\
\hline Otros & $\begin{array}{l}\text { Sal, aceite de oliva, vinagre, jugo de limón, salsa de } \\
\text { soya, cátsup, azúcar, miel, jalea, ate, almíbar, } \\
\text { mantequilla, gelatina }\end{array}$ & $\begin{array}{l}\text { Nueces, semillas, palomitas de maíz, coco rallado, } \\
\text { pepinillos, ajonjolí, mermelada, mostaza, humus }\end{array}$ \\
\hline
\end{tabular}

Adaptada de Murcio-Pérez, et al., $2012^{29}$.

emplearon dosis de hasta $20 \mathrm{mg}$ (superiores a la dosis de la formulación comercialmente disponible en México que contiene $10 \mathrm{mg}$ de BIS) ${ }^{33,34}$.

- PSCM. La preparación con PSCM es hiperosmolar, de bajo volumen y suele ser bien tolerada; sin embargo, debe evitarse en pacientes con nefropatía o cardiopatía, especialmente aquellos con arritmias cardiacas.

Un metaanálisis que comparó las preparaciones con PSCM y 4L PEG mediante un análisis de no inferioridad en la eficacia en la preparación, incluyendo calidad en la limpieza, TDA, TDP, eventos adversos y disposición para repetir la preparación a futuro, demostró que el PSCM no es inferior a 4L PEG en cuanto a efectividad.

No se encontraron diferencias en la TDP (RR: 0.94; IC 95\%: 0.82-1.08; $p=0.37$ ) o en la TDA (RR: 0.88; IC 95\%: 0.74-1.05; $p=0.16$ ). Una mayor proporción de pacientes refirió mayor satisfacción con la preparación de PSCM y se asoció con menos eventos adversos (RR: 0.78; IC 95\%: 0.66-0.93; $p=0.004)^{36}$. El uso de PSCM es una alternativa con efectividad semejante a $4 \mathrm{~L}$ PEG, pero es mejor tolerado.
Consideramos que, debido a su eficacia y adecuada tolerancia, 2L PEG + BIS o el PSCM pueden ser buenas alternativas en la preparación intestinal previa a colonoscopia.

LAS PREPARACIONES A BASE DE FOSFATO DE SODIO SE ASOCIAN A COMPLICACIONES RENALES E HIDROELECTROLÍTICAS

Recomendación fuerte. Nivel de evidencia moderado (GRADE 1B)

El NaP es una solución hiperosmótica de bajo volumen que atrae fluido hacia la luz del colon desde el espacio intravascular favoreciendo la peristalsis y la evacuación, sin embargo, los fosfatos que contiene pueden producir alteraciones electrolíticas y nefropatía aguda. Esto llevó a la Administración Federal de Fármacos (FDA) a emitir una advertencia sobre su prescripción $^{37}$.

Comparado con el PEG, el uso de $\mathrm{NaP}$ se asocia con mayor frecuencia a alteraciones hidroelectrolíticas, siendo las más frecuentes hiperfosfatemia, hipernatremia, hipocalcemia e hipocalemia ${ }^{38}$. 
En la actualidad, no se recomienda el $\mathrm{NaP}$ en pacientes con insuficiencia renal (depuración de creatinina $<60 \mathrm{ml} / \mathrm{min} / 1.73 \mathrm{~m}^{2}$ ), alteraciones electrolíticas preexistentes, insuficiencia cardiaca congestiva (clase III o IV de la New York Heart Association o fracción de eyección del ventrículo izquierdo $<50 \%$ ), cirrosis o ascitis, así como en aquellos con ingesta de inhibidores de la enzima convertidora de angiotensina, antagonistas de los receptores de la angiotensina II o antiinflamatorios no esteroideos. Se desaconseja su uso en niños o adolescentes menores de 18 años $^{39,40}$.

La AMEG no recomienda su uso en la preparación para colonoscopia.

\section{EL MÉTODO DE PREPARACIÓN EN DOSIS DIVIDIDA INCREMENTA LA FRECUENCIA DE PACIENTES CON PREPARACIÓN ADECUADA}

Recomendación fuerte. Nivel de evidencia alto (GRADE 1A)

Debido a que la tolerancia de la preparación $4 \mathrm{~L}$ PEG esta limitada por la necesidad de ingerir un alto volumen, la dosis dividida se ha convertido en una buena alternativa. Esta última consiste en dar una porción de la preparación (habitualmente la mitad) el día previo al procedimiento y el resto de la preparación el día de la colonoscopia.

Comparada con la preparación de 4L PEG en dosis única, la dosis dividida aumentó significativamente la frecuencia de procedimientos con preparación satisfactoria (RM: 3.7; IC 95\%: 2.79-4.91; $p<0.01$ ), así como la disposición para repetir la preparación (RM: 1.76). Se asoció a menos eventos adversos (náuseas RM: $0.55)$ y a menos casos de preparación incompleta (RM: $0.53)^{41}$.

Las ventajas observadas con la dosis dividida de PEG se han observado también con el uso de $\mathrm{NaP}$ y picosulfato de sodio. La administración de estas en dosis divididas logra mejores puntajes de limpieza global (RM: 2.51; IC 95\%: 1.86-3.39) en un mayor número de pacientes. Se han observado mejores resultados con NaP (RM: 9.34), seguido del picosulfato (RM: 3.54) y PEG (RM: 2.60). Usando dosis dividida, un mayor número de pacientes completa adecuadamente la preparación y está dispuesto a repetirla (RM: 1.9), particularmente aquellas de bajo volumen (RM: 4.95$)^{42}$. Es importante que la colonoscopia se realice dentro de las cinco horas siguientes a completar la ingesta de la segunda parte de la preparación ${ }^{43}$.

Cuando el procedimiento de colonoscopia se realizará por la tarde, la ingesta de la preparación completa el mismo día del procedimiento puede ser una alternativa válida comparado con la preparación dividida, ya que no se han reportado diferencias significativas en el número de pacientes que logran una limpieza adecuada ( 85.3 vs. $86.3 \%$ respectivamente), a pesar de que el cumplimiento del tratamiento es mayor en aquellos que siguen una preparación dividida (89.7 vs. $96.6 \%)^{44}$.

Recomendamos el uso de dosis dividida, independientemente del compuesto utilizado, pues ha demostrado ser una estrategia que mejora la calidad de la limpieza e incrementa la tolerancia del paciente ${ }^{11,45}$.

\section{EL TIEMPO ENTRE EL FINAL DE LA PREPARACIÓN Y EL INICIO DEL PROCEDIMIENTO NO DEBE EXCEDER 8 HORAS}

Recomendación fuerte. Nivel de evidencia alto (GRADE 1A)

El tiempo de la preparación intestinal para la colonoscopia influye en la calidad de la limpieza del intestino y en el éxito del procedimiento. Se ha visto que la dosis dividida ha dado mejores resultados en la calidad de la colonoscopia en comparación con la dosis completa un día previo.

La segunda porción de la preparación o la última dosis debe administrarse de tres a ocho horas antes del inicio de la colonoscopia. Siddiqui, et al. reportaron que después de un lapso de ocho horas de concluida la preparación, por cada hora adicional entre el final de la preparación intestinal y la colonoscopia, la posibilidad de lograr una buena o excelente limpieza en el colon derecho disminuye hasta en un $10 \%{ }^{46}$.

La Sociedad Americana de Anestesiología recomienda ayuno de líquidos claros de dos horas previo a la sedación del paciente, por lo que es necesario que los pacientes completen la preparación por lo menos dos horas antes del procedimiento ${ }^{11}$. Un estudio prospectivo en el que compararon la dosis dividida y la dosis completa el día previo al estudio no encontró diferencias en la cantidad de líquido gástrico residual ${ }^{47}$.

\section{EL USO DE PROCINÉTICOS NO MEJORA LA CALIDAD DE LA PREPARACIÓN PARA LA COLONOSCOPIA}

Recomendación débil. Nivel de evidencia moderado (GRADE 2B)

La administración concomitante de procinéticos a las preparaciones intestinales ha sido evaluada en estudios controlados. La metoclopramida, un antagonista de la dopamina que aumenta la amplitud de la contracción 
gástrica e incrementa la peristalsis del duodeno y yeyuno no modifica la motilidad colónica ${ }^{48}$.

Diversos estudios clínicos aleatorizados demostraron que la adición de metoclopramida, cisaprida, tegaserod, mosaprida o itoprida a la preparación no mejora la calidad de la limpieza intestinal y tampoco su tolerancia ${ }^{48-52}$.

No se recomienda el uso de procinéticos como parte del esquema habitual de preparación intestinal para colonoscopia.

En la tabla 2 se resumen las principales características, ventajas y desventajas de las principales alternativas para la preparación colónica ${ }^{31,40,53-55}$.

\section{Técnica de la colonoscopia, sobre la tasa de intubación cecal y dolor posprocedimiento}

\section{La tasa de intubación cecal y visualización de sus identificadores anatómicos debe ser superior al $90 \%$ y siempre debe documentarse fotográficamente}

Recomendación fuerte. Nivel de evidencia moderado (GRADE 1B)

La intubación cecal se define como la llegada de la punta del endoscopio a un punto proximal a la válvula ileocecal, de tal manera que todas las paredes del ciego, la confluencia de las tenias colónicas («pata de gallo»), el orificio apendicular y los labios de la válvula ileocecal se vean claramente.

La intubación cecal permite identificar las neoplasias colorrectales que se localizan en el colon proximal incluyendo el ciego, por lo que tasas bajas de intubación cecal se asocian a tasas elevadas de cáncer de intervalo en zonas proximales del colon ${ }^{56}$.

La intubación cecal debe documentarse fotográficamente en todos los casos e idealmente incluir el íleon terminal. Esta se confirma al identificar las estructuras anatómicas distintivas del ciego, como los labios o el orificio de la válvula ileocecal, orificio apendicular (si no hay antecedentes de apendicectomía), confluencia de las tenias y/o bien intubar el íleon terminal ${ }^{57-59}$. La tasa de intubación cecal ideal es $>90 \%$ de los casos generales y > 95\% cuando la indicación es escrutinio de CCR en adultos sanos ${ }^{60}$.

Cuando la indicación de la colonoscopia no es escrutinio de CCR y/o bien el procedimiento se aborta por mala preparación, colitis grave o porque la indicación es el tratamiento de una estenosis o un pólipo distal al ciego, la falta de fotodocumentación de las referencias anatómicas antes descritas no debe considerarse en la evaluación de los indicadores de calidad, pero si debe fotodocumentarse la causa por la que se decidió interrumpir el procedimiento.

En la actualidad se sugiere que el íleon terminal sea intubado en la mayor parte de los casos y se considera obligatorio en casos con enfermedad inflamatoria intestinal, diarrea crónica, hemorragia de intestino delgado y extracción de cuerpos extraños de íleon.

Existen técnicas que incrementan la tasa de intubación cecal que se discutirán brevemente a continuación.

LA TASA DE INSERCIÓN CECAL UTILIZANDO LA TÉCNICA DE INSERCIÓN ASISTIDA POR UN OPERADOR ES SIMILAR A LA OBTENIDA POR LA TÉCNICA DE DOS OPERADORES

Recomendación débil. Nivel de evidencia bajo (GRADE 2C)

La técnica de un operador es recomendada por las sociedades profesionales y representa una práctica habitual en los EE.UU.61, aunque en algunos países europeos y orientales aún se utiliza la técnica de dos operadores.

Diferentes estudios no encontraron diferencias en la tasa de intubación cecal, TDA y tiempo de retirada; en dos el tiempo de inserción fue menor con un operador y solo en uno la inserción con dos operadores fue más rápida ${ }^{62-64}$.

Aun cuando la colonoscopia con uno o dos operadores son comparables, la técnica de un operador requiere menos personal. La evidencia actual no justifica cambiar la técnica que se utilice habitualmente.

LA INMERSIÓN EN AGUA Y EL RECAMBIO DE AGUA SON TÉCNICAS QUE INCREMENTAN EL ÍNDICE DE INTUBACIÓN CECAL (COLONOSCOPIAS COMPLETAS)

Recomendación fuerte. Nivel de evidencia moderado (GRADE 1B)

La inmersión en agua consiste en la distensión del colon con infusión de agua mediante una bomba, hasta lograr la intubación cecal; el líquido se aspira al momento de la retirada.

El recambio de aguda infunde agua alternando con su aspiración junto con residuos fecales y burbujas durante el avance.

Cuatro metaanálisis, uno de ellos con gran heterogeneidad de los estudios incluidos, encontraron que el recambio de agua toma más tiempo de duración del 
Tabla 2. Características de los diferentes tipos de preparación para colonoscopia

\begin{tabular}{|c|c|c|c|c|}
\hline $\begin{array}{l}\text { Tipo de } \\
\text { preparación }\end{array}$ & Osmolaridad & Ventajas & Desventajas & Observaciones \\
\hline PEG 4L & Isoosmolar & $\begin{array}{l}\text { Eficacia } \\
\text { Seguridad } \\
\text { No altera el } \\
\text { aspecto } \\
\text { endoscópico ni } \\
\text { histológico de la } \\
\text { mucosa de colon }\end{array}$ & $\begin{array}{l}\text { Intolerancia por alto volumen } \\
\text { Ingesta incompleta en el } \\
15-20 \% \text { de los pacientes } \\
\text { Menor disposición a repetir } \\
\text { el esquema a futuro }\end{array}$ & $\begin{array}{l}\text { Considerada preparación estándar por eficacia y } \\
\text { seguridad } \\
\text { No suele asociarse con deshidratación o } \\
\text { desequilibrio hidroelectrolítico } \\
\text { Puede utilizarse en pacientes con hepatopatía, } \\
\text { nefropatía, insuficiencia cardiaca y población } \\
\text { geriátrica y pediátrica } \\
\text { Preparación de elección en pacientes con } \\
\text { hemorragia de tubo digestivo bajo y en } \\
\text { pacientes con enfermedad inflamatoria intestinal } \\
\text { En dosis dividida mejora considerablemente su } \\
\text { tolerancia } \\
\text { Puede utilizarse en el embarazo (segundo } \\
\text { trimestre), valorando riesgo-beneficio }\end{array}$ \\
\hline PEG 2L & Isoosmolar & $\begin{array}{l}\text { Eficacia } \\
\text { Seguridad } \\
\text { Bajo volumen } \\
\text { Bien tolerada }\end{array}$ & $\begin{array}{l}\text { Su combinación con } \\
\text { bisacodilo a dosis altas } \\
\text { puede asociarse con colitis } \\
\text { isquémica }\end{array}$ & $\begin{array}{l}\text { Mantiene perfil de seguridad y eficacia de PEG } 4 \mathrm{~L} \\
\text { Se puede combinar con laxantes para aumentar } \\
\text { su eficacia } \\
\text { Debe evitarse la combinación con ácido } \\
\text { ascórbico en pacientes con deficiencia de G6PDH } \\
\text { Ideal en pacientes con cirugía gástrica restrictiva }\end{array}$ \\
\hline $\begin{array}{l}\text { Picosulfato } \\
\text { de sodio y } \\
\text { citrato de } \\
\text { magnesio }\end{array}$ & Hiperosmolar & $\begin{array}{l}\text { Eficacia } \\
\text { Bajo volumen } \\
\text { Buen sabor }\end{array}$ & $\begin{array}{l}\text { Hiponatremia } \\
\text { Hipermagnesemia }\end{array}$ & $\begin{array}{l}\text { Deshidratación si no se ingiere suficiente agua } \\
\text { adicional } \\
\text { Debe evitarse en pacientes con enfermedad renal } \\
\text { El citrato de magnesio se asocia con hipotensión } \\
\text { y bradicardia } \\
\text { Puede causar lesiones mucosas que confunde } \\
\text { con enfermedad inflamatoria intestinal }\end{array}$ \\
\hline $\begin{array}{l}\text { Fosfato de } \\
\text { sodio }\end{array}$ & Hiperosmolar & $\begin{array}{l}\text { Eficacia } \\
\text { Adecuada } \\
\text { tolerancia }\end{array}$ & $\begin{array}{l}\text { Inadecuado perfil de } \\
\text { seguridad } \\
\text { Asociado con desarrollo de } \\
\text { insuficiencia renal: nefropatía } \\
\text { aguda por fosfatos }\end{array}$ & $\begin{array}{l}\text { No se recomienda en enfermedad renal, } \\
\text { insuficiencia cardiaca o hepática, población } \\
\text { pediátrica o geriátrica } \\
\text { Evitar en enfermedad inflamatoria intestinal } \\
\text { Interacciones con diuréticos, inhibidores de la } \\
\text { ECA, antagonistas ARA II }\end{array}$ \\
\hline
\end{tabular}

PEG: polietilenglicol; G6PDH: glucosa-6-fosfato deshidrogenasa; ECA: enzima convertidora de la angiotensina; ARA II: antagonistas de los receptores de la angiotensina II. Adaptada de Johnson, et al., 201440; Parekh, et al., 201931; Ruiz-Romero, et al., 201653; Parra-Blanco, et al., 201454; Sharara, et al., $2103^{55}$.

estudio comparada con la inmersión y con la insuflación de aire. Algunos reportaron una mejor TDA y tasa de intubación cecal (incluyendo pacientes considerados técnicamente difíciles) comparada con la insuflación de dióxido de carbono $\left(\mathrm{CO}_{2}\right)$ y la inmersión y en otros las tres técnicas fueron comparables. Tampoco hubo diferencias en molestias o dolor posterior al procedimiento y solo dos metaanálisis favorecieron al recambio de agua ${ }^{65-68}$.

Con la evidencia actual no se puede recomendar el uso de una técnica específica. Se requieren más estudios que demuestren claramente si las técnicas mencionadas son comparables en todos los desenlaces 0 si efectivamente la técnica de recambio de agua mejora la tasa de intubación cecal, la TDA y se asocia a menor dolor.

\section{EL USO DE RIGIDEZ VARIABLE NO MEJORA LA TASA DE INTUBACIÓN CECAL, NO DISMINUYE EL TIEMPO DE INSERCIÓN, PERO PARECE DISMINUIR EL DOLOR POSTERIOR AL PROCEDIMIENTO}

\section{Recomendación débil. Nivel de evidencia bajo (GRADE 2C)}

Los colonoscopios con rigidez variable (RV) cuentan con un dispositivo que permite ajustar durante el procedimiento la rigidez estructural del colonoscopio en tres diferentes grados. Se sugiere usar el modo más flexible para franquear las angulaciones del sigmoides y posteriormente incrementar la rigidez para reducir las asas en colon transverso y derecho. La evidencia sobre las potenciales ventajas de la RV es limitada y contradictoria al compararse con colonoscopios sin RV. No se han 
encontrado diferencias en la tasa de intubación cecal, tiempo de inserción ni tiempo total del procedimiento. En pacientes con antecedente de cirugía abdominal, el colonoscopio pediátrico con RV se asocia a menos dolor comparado con el de colonoscopio convencional normal, además que se necesitaron menos maniobras auxiliares $^{69,70}$; algunos estudios indican que el colonoscopio con RV se asocia a menos dolor posprocedimiento y menor requerimiento de sedantes ${ }^{71,72}$.

Solo un metaanálisis reportó mejores tasas de intubación cecal con RV comparada con colonoscopio tradicional (RM: 2.08; IC 95\%:1.29-3.36). Sin embargo, se debe mencionar que los estudios incluidos no fueron cegados y el uso de RV no fue controlado ${ }^{72}$.

\section{EL USO DE UN CAPUCHÓN TRANSPARENTE DURANTE LA COLONOSCOPIA MEJORA EL TIEMPO DE INSERCIÓN}

Recomendación fuerte. Nivel de evidencia moderado (GRADE 1B)

El capuchón de plástico transparente (CAP) es un dispositivo que se coloca en la punta del colonoscopio; este permite una mejor visualización de la mucosa, ayuda a reducir la formación de asas y comprime los pliegues semilunares, lo que disminuye puntos ciegos $^{73}$. Un metaanálisis de Cochrane encontró que la colonoscopia con CAP disminuye marginalmente el tiempo de intubación cecal, mejora la TDP y produce menos dolor comparado con la colonoscopia estándar. Sin embargo, no se reporta información sobre la tasa de intubación cecal ${ }^{74}$.

\section{Dolor posprocedimiento}

Como se mencionó previamente, parámetros como la TDA, la velocidad, facilidad y frecuencia con la que se llega al ciego y la máxima visualización de mucosa son índices útiles para evaluar la calidad del procedimiento. Otros como el índice de complicaciones tardías o presencia de dolor/incomodidad del paciente posterior al procedimiento son indicadores de calidad igualmente importantes. Se han desarrollado técnicas para disminuir complicaciones y dolor posterior a una colonoscopia.

\section{LA INSUFLACIÓN CON DIÓXIDO DE CARBONO DURANTE UNA COLONOSCOPIA DISMINUYE LAS MOLESTIAS POSTERIORES AL PROCEDIMIENTO}

Recomendación fuerte. Nivel de evidencia alto (GRADE 1A)
El aire insuflado durante una colonoscopia permanece tiempo prolongado en el interior del colon debido a sus bajas tasas de depuración, difusión y absorción. Esto provoca distensión, dolor y malestar abdominal incluso hasta seis horas después del procedimiento.

$\mathrm{EI} \mathrm{CO}_{2}$ es un gas que se depura rápidamente de los intestinos mediante difusión pasiva. La insuflación con $\mathrm{CO}_{2}$ se asocia a menor frecuencia e intensidad de dolor, distensión y malestar abdominal posterior a una colonoscopia ${ }^{75-78}$.

Su uso generalizado fue limitado por el temor a efectos adversos relacionados con la retención de $\mathrm{CO}_{2}{ }^{79}$ pero diversos estudios han demostrado que su uso es seguro 80,81 .

Un metaanálisis que utilizó un modelo de efectos aleatorios debido a la heterogeneidad encontrada en algunos de los desenlaces mayores encontró que la insuflación de $\mathrm{CO}_{2}$ disminuye significativamente el dolor durante (RR: 0.77; IC 95\%: 0.62-0.96), 1 hora (RR: 0.26; IC 95\%: 0.16-0.46), 6 horas (RR: 0.36; IC95\%: 0.200.64) y 24 horas (RR: 0.53; IC 95\%: 0.31-0.91) posterior al procedimiento endoscópico. El número necesario que tratar (NNT) para lograr estos efectos durante el procedimiento, así como 1, 6 y 24 horas posterior a este fue 7, 2, 3 y 12 pacientes respectivamente. Al comparar la insuflación de aire con la de $\mathrm{CO}_{2}$ no se encontraron diferencias en el perfil de seguridad, efectos adversos, volumen administrado e intubación cecal ${ }^{82}$.

Las dos poblaciones en riesgo de retención de $\mathrm{CO}_{2}$ son aquellos con enfermedad pulmonar obstructiva crónica y quienes reciben sedación. Aunque se ha estimado un riesgo bajo de retención grave con la cantidad de $\mathrm{CO}_{2}$ insuflado durante la colonoscopia, esta información es escasa y de baja calidad.

Un solo estudio demostró elevación no significativa de la presión parcial de dióxido de carbono $\left(\mathrm{pCO}^{2}\right)$ posterior al procedimiento, la cual no superó los limites normales ${ }^{83}$. Se requieren ensayos clínicos controlados que midan la $\mathrm{pCO}_{2}$ y/o el volumen corriente final de $\mathrm{CO}_{2}$ (capnografía) en pacientes bajo sedación y/o con enfermedad pulmonar obstructiva crónica para establecer con mayor certeza el perfil de seguridad de la insuflación de $\mathrm{CO}_{2}$. En la actualidad la indicación de insuflación con $\mathrm{CO}_{2}$ debe individualizarse ${ }^{84}$.

\section{LA INSERCIÓN DEL COLONOSCOPIO EN POSICIONES DISTINTAS AL DECÚBITO LATERAL IZQUIERDO MEJORAN EL TIEMPO DE INSERCIÓN Y LA TOLERANCIA DEL ESTUDIO EN POBLACIONES ESPECIALES}

Recomendación débil. Nivel de evidencia bajo (GRADE 1C) 
La inserción del colonoscopio consume tiempo y puede asociarse a complicaciones serias (p. ej., perforación). El decúbito lateral izquierdo (DLI) facilita la inserción y es la posición sugerida para realizar el procedimiento, sin embargo, hay escasos estudios clínicos que lo demuestren.

En pacientes sometidos a colonoscopia sin sedación el tiempo de intubación cecal (280 vs.339 segundos; $p<0.001)$ y la frecuencia e intensidad de dolor $(3.4 \pm$ 1.6 vs. $4.0 \pm 1.7 ; p=0.02$ ) fueron menores cuando la colonoscopia se realizó en decúbito supino (DS) comparado con DLI ${ }^{85}$. Resultados similares se observaron cuando la colonoscopia se realiza en decúbito lateral derecho (DLD). Comparado con DLI, el tiempo promedio de inserción al ciego fue menor $(p=0.0078)$. Los pacientes más beneficiados son mujeres y aquellos con cirugía abdominal previa.

Aunque la evidencia es limitada, el realizar una colonoscopia en DS o DLD ofrece beneficios y mejora el tiempo de inserción y tolerancia especialmente en mujeres y pacientes con cirugía abdominal previa ${ }^{86}$.

\section{EL USO DE FLUOROSCOPIA O DE DISPOSITIVOS VIRTUALES DE NAVEGACIÓN Y SIMULACIÓN NO MEJORA LOS ÍNDICES DE CALIDAD}

Recomendación débil. Nivel de evidencia bajo (GRADE 2C)

Considerando que muchos de los índices de calidad en colonoscopia están relacionados con la técnica, se ha sugerido que el uso de dispositivos como inserción guiada por fluoroscopia, simuladores y sistemas de navegación virtual, entre otros, pueden tener un impacto positivo en el desarrollo y mejora de las habilidades psicomotrices, lo que resultaría en procedimientos de mayor calidad. Sin embargo, la información científica disponible es limitada y de baja calidad, por lo que no se recomienda el uso de estos, excepto en protocolos de investigación y como métodos adicionales a la enseñanza habitual ${ }^{58,87-91}$.

\section{Tasa de detección de adenomas}

\section{La colonoscopia es el estándar de oro para la detección y resección de pólipos y adenomas en el colon}

Recomendación fuerte. Nivel de evidencia alto (GRADE 1A)

La colonoscopia se considera el estándar de oro para la detección y tratamiento de adenomas, neoplasias incipientes y avanzadas. Tiene una sensibilidad para detectar adenomas avanzados del 90\%, para adenomas pequeños $(<1 \mathrm{~cm})$ del $75 \%$ y para CCR del $94.7 \%{ }^{92,93}$.

La colonoscopia, adicionalmente al diagnóstico visual, permite la obtención de tejido mediante biopsias y la resección de lesiones (p. ej., polipectomía). La colonoscopia evita el desarrollo de cáncer avanzado y reduce significativamente la incidencia y mortalidad asociadas a CCR hasta en el 58 y $64 \%$ respectivamente ${ }^{94}$.

\section{La tasa de detección de adenomas > 25\% es un parámetro de calidad en colonoscopia de escrutinio}

Recomendación fuerte. Nivel de evidencia alto (GRADE 1A)

La TDA se estima dividiendo el número de colonoscopias en que se identifican adenomas confirmados histológicamente, entre el número total de colonoscopias de escrutinio realizadas.

La TDA evalúa la habilidad del endoscopista en los programas de escrutinio de CCR y es un factor predictor independiente de cáncer de intervalo. Esta correlaciona inversamente con el CCR de intervalo (CCR después de colonoscopia) y con la mortalidad asociada a CCR.

La TDA esperada en poblaciones occidentales durante programas de escrutinio de CCR debe ser al menos del 20 y el $40 \%$ cuando se utiliza prueba inmunológica de sangre en heces como herramienta inicial de escrutinio. Actualmente la Sociedad Americana de Endoscopia Gastrointestinal (ASGE) y otras guías de calidad en colonoscopia recomiendan alcanzar un mínimo de TDA del $30 \%$ en hombres y el $20 \%$ en mujeres ${ }^{5,11}, 95-97$.

No existe información sobre la TDA promedio y su asociación con CCR de intervalo en nuestro país, por lo que proponemos un valor de TDA mayor o igual al $25 \%$ como parámetro para evaluar la calidad de colonoscopia en programas de escrutinio.

Se sugiere que cada colonoscopista conozca su TDA y procure incrementarla y/o mantenerla en los parámetros sugeridos. Existen técnicas que pueden incrementar la TDA, las cuales se describen brevemente a continuación.

LA COLONOSCOPIA DE INMERSIÓN NO INCREMENTA LA TASA DE DETECCIÓN DE ADENOMAS, AUNQUE MEJORA LA CARACTERIZACIÓN DE ESTOS

Recomendación fuerte. Nivel de evidencia bajo (GRADE 1C) 
LA COLONOSCOPIA CON TÉCNICA DE RECAMBIO DE AGUA INCREMENTA LA TASA DE DETECCIÓN DE ADENOMAS

Recomendación débil. Nivel de evidencia bajo (GRADE 2C)

De acuerdo con reportes de Leung, et al., la TDA obtenida mediante colonoscopia por inmersión es similar a la obtenida mediante colonoscopia estándar con insuflación con aire ${ }^{98}$.

Sin embargo, la técnica de recambio de agua comparada con el método tradicional con aire se asocia a un incremento en la TDA de hasta el 10\% ${ }^{99-101}$.

Ambas técnicas tienen la ventaja de mejorar la visión, al eliminar todo el contenido del colon, disminuir las molestias y/o dolor posterior al procedimiento, pero tienen la desventaja de incrementar la duración de este $^{98}$.

\section{LA UTILIZACIÓN DE DISPOSITIVOS QUE DEPRIMEN LOS \\ PLIEGUES COLÓNICOS (CAPUCHÓN PLÁSTICO, ENDOCUFF VISION ${ }^{\mathrm{TM}}$ ) Y MANIOBRAS ESPECIALES (RETROFLEXIÓN CECAL) INCREMENTAN LA TASA DE DETECCIÓN DE ADENOMAS}

Recomendación débil. Nivel de evidencia bajo (GRADE 2C)

La efectividad de la colonoscopia de escrutinio, como se mencionó previamente, depende de una adecuada TDA, lo cual depende de la limpieza intestinal y del porcentaje de área mucosa examinada.

Es importante considerar que el objetivo principal es hacer una revisión minuciosa de la mucosa en toda su extensión. Esto se logra con una preparación óptima, tiempo y sistematización de la revisión.

Los dispositivos que incrementen el campo visual, ya sea deprimiendo los pliegues colónicos, incrementando el campo visual con un mayor número de cámaras o bien mediante maniobras especiales, pueden incrementar la TDA.

El uso de un CAP adherido a la punta del endoscópico incrementa la TDA en comparación con las colonoscopias convencionales (47.0 vs. $42.6 \%)$. Específicamente, incrementa la detección de pólipos $<5 \mathrm{~mm}$ y también disminuye el tiempo de intubación cecal ${ }^{102,103}$.

El Endocuff Vision ${ }^{\mathrm{TM}}$ es un dispositivo que se coloca en la punta distal del colonoscopio que contiene una fila de brazos flexibles que dan vuelta y aplanan los pliegues del colon, mejorando la visión, ha demostrado incrementar la detección de adenomas comparado con la colonoscopia convencional con un NNT de 19. El mayor beneficio se ha observado en endoscopistas con TDA < 35\%104,105.

Realizar retroflexión en el colon derecho o bien una segunda observación incrementa la TDA entre un 6 y un $10 \%(33.6 \text { vs. } 26.7 \% \text {; } p<0.01)^{106}$.

Existen tecnologías en desarrollo que incorporan más cámaras y globos en la punta distal del colonoscopio, con el objetivo de incrementar el área de mucosa examinada, las cuales aún están en evaluación, por lo que su disponibilidad y utilidad son limitadas e inciertas ${ }^{107}$.

\section{LA TASA DE DETECCIÓN DE ADENOMAS SE CORRELACIONA DE MANERA DIRECTAMENTE PROPORCIONAL CON EL GRADO DE LIMPIEZA INTESTINAL}

Recomendación fuerte. Nivel de evidencia moderado (GRADE 1B)

Una adecuada limpieza intestinal está asociada con una alta TDA ${ }^{6,108}$. Una preparación de baja calidad reduce la eficacia de la colonoscopia hasta en un $20 \%$ y reduce significativamente la detección de adenomas tempranos (RM: 0.53; IC 95\%: 0.46-0.62; $p<0.001)^{109}$.

\section{EL TIPO DE SEDACIÓN (MODERADA O PROFUNDA) NO MODIFICA LA TASA DE DETECCIÓN DE ADENOMAS}

Recomendación fuerte. Nivel de evidencia bajo (GRADE 1C)

El propofol, que proporciona una sedación profunda con una inducción y recuperación rápida, ha desplazado a la sedación moderada que se realizaba mediante la administración de una benzodiazepina y un opioide ${ }^{110,111 .}$

El uso de sedación durante la colonoscopia, además de facilitar el procedimiento y disminuir las molestias al paciente, incrementa la tolerabilidad del estudio, la tasa de intubación cecal y en algunos estudios ha demostrado mejorar la TDP ${ }^{112-114}$. Sin embargo, estudios recientes indican que el tipo de sedación (moderada o profunda) no modifica la TDA ni laTDP112,114,115.

La mayoría de los estudios disponibles son retrospectivos realizados en un solo centro, por lo que es necesario que se realicen estudios aleatorizados controlados con un mayor número de casos que permitan determinar el impacto del tipo de sedación sobre la TDA y la TDP. 


\section{LA ENDOSCOPIA DE ALTA RESOLUCIÓN MEJORA LA TASA DE DETECCIÓN DE ADENOMAS}

Recomendación fuerte. Nivel de evidencia alto (GRADE 1A)

Aproximadamente el $30.2 \%$ de la población mayor de 50 años, asintomática y de riesgo promedio, tendrá un adenoma colorrectal (ACR) en una colonoscopia de escrutinio y el 6-10\% tendrán un adenoma avanzado ${ }^{116}$. El $80-90 \%$ de los ACR son menores de $1 \mathrm{~cm}$ y del $0.2-$ $11 \%$ serán pólipos malignos. Se estima que durante una colonoscopia de escrutinio, la tasa de pérdida de adenomas es del 22\% (IC 95\%: 15-32\%), y la tasa de pérdida de adenomas avanzados es del $5.4-11 \%{ }^{117}$.

El uso de técnicas y dispositivos como equipos de alta definición y cromoendoscopia virtual, además de los ya comentados (CAP, Endocuff Vision ${ }^{\mathrm{TM}}$, inmersión en agua, retroflexión, etc.), ayudan a incrementar la TDA $^{118}$.

Los endoscopios de alta definición comparados con aquellos de luz blanca son superiores en la detección de adenomas planos ${ }^{119}$. La alta definición incrementa la TDP, en el 3.8\% (IC 95\%: 1-6.7\%) con un NNT de 26 y la TDA en un 3.5\% (IC 95\%: 0.9-6.1\%) con NNT de 28, especialmente de adenomas pequeños. Hasta el momento no se han reportado diferencias en la identificación de adenomas avanzados ${ }^{120}$.

\section{LA CROMOENDOSCOPIA VIRTUAL Y/O CONVENCIONAL NO MEJORA LA TASA DE DETECCIÓN DE ADENOMAS}

Recomendación fuerte. Nivel de evidencia de alto (GRADE 1A)

La cromoendoscopia virtual no mejora los índices de detección de adenomas, pero sí la caracterización de los pólipos. Esta técnica ayuda a distinguir un pólipo neoplásico de uno no neoplásico con una sensibilidad del 90 al $91 \%$ y especificidad del 85 al $88 \%$, según el equipo utilizado. La cromoendoscopia convencional mostró rendimiento diagnóstico similar ${ }^{121,122}$.

Esta técnica aún no reemplaza al estudio histopatológico, por lo que en la actualidad se recomienda extirpar y enviar a estudio patológico todos los pólipos observados.

LAS PRUEBAS DE ESCRUTINIO NO INVASORAS (SANGRE OCULTA EN HECES) NO INCREMENTAN LA TASA DE DETECCIÓN DE ADENOMAS

Recomendación fuerte. Nivel de evidencia alto (GRADE 1A)
La evidencia actual indica que la detección de sangre oculta en heces positiva se asocia con la presencia de lesiones avanzadas de CCR, pero no incrementan la TDA global'123.

La prueba inmunohistoquímica fecal (FIT) y de ADN fecal son superiores a la prueba de guayaco en el diagnóstico de $\mathrm{CCR}^{124,125}$. Interesantemente, una prueba FIT positiva seguida de colonoscopia incrementa la TDA y el número de adenomas encontrados, especialmente de lesiones avanzadas comparado con colonoscopia de escrutinio sin FIT previa. Esta puede ayudar a seleccionar pacientes que se beneficiarán de una colonoscopia en programas donde el acceso a esta es restringido ${ }^{124}$.

\section{Polipectomía. Resección completa y prevención de complicaciones}

Todos los pólipos identificados deben ser resecados en su totalidad sin importar su tamaño. Se sugiere analizar y reportar el aspecto macroscópico, morfología, predictores de invasión, tamaño, etc., y así poder elegir el mejor método de resección.

La tasa de recuperación de pólipos debe ser mayor del $80 \%$ para pólipos < $10 \mathrm{~mm}$ y mayor del $95 \%$ para pólipos $>10 \mathrm{~mm}$.

Los pólipos diminutos (menores de $5 \mathrm{~mm}$ ) pueden ser extirpados con asa fría (AF); en aquellos que midan entre 1 y $3 \mathrm{~mm}$ se puede utilizar una pinza de biopsia fría. Los pólipos pequeños de 6 a $9 \mathrm{~mm}$ idealmente deberán extirparse con $\mathrm{AF}$, pues tiene un mejor perfil de seguridad comparado con asa caliente (AC). Los pólipos sésiles de 10-19 $\mathrm{mm}$ se sugiere que sean tratados con AC, previa inyección de la submucosa ${ }^{93-97}$.

A continuación se describe brevemente algunas técnicas que se asocian a las mejores tasas de resección completa y menos complicaciones, aunque la elección de estas deberá individualizarse y dependerá también del material disponible y experiencia de cada endoscopista.

\section{Resección completa}

LA POLIPECTOMÍA CON ASA FRÍA DE PÓLIPOS DIMINUTOS (< 5 MM) Y PARA PÓLIPOS SÉSILES DE 6 a 9 MM LOGRA TASAS DE RESECCIÓN COMPLETA SUPERIORES A LAS LOGRADAS POR LA PINZA DE BIOPSIA FRÍA CONVENCIONAL

Recomendación fuerte. Nivel de evidencia alto (GRADE 1A) 
La polipectomía con pinza fría jumbo logra tasas de resección completa similares a la logradas con asa fría

Recomendación débil. Nivel de evidencia moderado (GRADE 2B)

Diferentes estudios han demostrado que la resección con AF es superior a la polipectomía con pinza fría convencional en relación con la resección completa de pólipos diminutos ${ }^{126-129}$ y tiene un promedio de resección completa similar a la polipectomía con AC (94 vs. $95 \% ; p=0.31)^{130-132}$, además de que se evitan los daños térmicos del electrocauterio.

Comparada con la pinza fría, el AF logra la resección completa en el $99.4 \%$ con una tasa de hemorragia inmediata y tardía del 0.8 y $0.2 \%$ respectivamente. La recurrencia local ocurre en el $2.1 \%$ de los casos a un año de seguimiento, especialmente de las lesiones $>3 \mathrm{~mm}^{133}$.

Comparada con la polipectomía con pinza fría jumbo (PFJ) el promedio de resección completa fue similar (PFJ: 92.0 vs. AF: 92.2\%; $p=0.94$ ), al igual que el tiempo del procedimiento, el promedio de retirada de la muestra y el porcentaje de eventos adversos pospolipectomía ${ }^{134}$.

\section{LA POLIPECTOMÍA CON ASA CALIENTE DE PÓLIPOS SÉSILES DE 10 A 19 MM CON O SIN INYECCIÓN DE LA SUBMUCOSA LOGRA TASAS DE RESECCIÓN COMPLETA SATISFACTORIAS Y SIMILARES A LA RESECCIÓN ENDOSCÓPICA DE LA MUCOSA}

Recomendación fuerte. Nivel de evidencia bajo (GRADE 1C)

La polipectomía con asa fría de pólipos sésiles de 10 a $19 \mathrm{~mm}$ con o sin inyección de la submucosa logra tasas de resección completa similares a la resección con asa caliente

Recomendación fuerte. Nivel de evidencia bajo (GRADE 1C)

La polipectomía con $\mathrm{AC}$ es la técnica más utilizada para resecar pólipos de 10 a $19 \mathrm{~mm}$ de diámetro. La inyección de la submucosa aumenta la posibilidad de la resección en bloque y disminuye los riesgos de daño térmico profundo ${ }^{97}$.

El estudio Complete Adenoma Resection (CARE) reportó un promedio de resección completa de pólipos entre $10-19 \mathrm{~mm}$ con $\mathrm{AC}$ de $83.7 \%{ }^{135}$.
Comparada con la resección endoscópica de la mucosa (REM) en pólipos pequeños, sésiles o planos, ambas técnicas tienen tasas de resección completa similares (AC: $88.4 \%$ vs. REM: $92.8 \% ; p=0.2)^{136}$.

La polipectomía con AF de pólipos mayores de 10 mm logra una tasa de resección completa del 99.3\% (IC 95\%: 98.6-100\%) y se asocia a una frecuencia baja de eventos adversos 1.1\% (IC 95\%: 0.2-2.0\%; 12 : 0\%). El promedio de hemorragia intraprocedimiento y posprocedimiento reportado es del 0.7\% (IC 95\%: 0-1.4\%) y del $0.5 \%$ (IC 95\%: 0.1-1.2\%) respectivamente ${ }^{137}$.

\section{LA POLIPECTOMÍA CON ASA CALIENTE PREVIA} INYECCIÓN DE LA SUBMUCOSA Y/O COLOCACIÓN DE MÉTODOS HEMOSTÁTICOS MECÁNICOS EN PÓLIPOS MAYORES A 20 MM O CON TALLO MAYOR A 10 MM DE DIÁMETRO DISMINUYE LA TASA DE COMPLICACIONES (PERFORACIÓN, HEMORRAGIA)

Recomendación fuerte. Nivel de evidencia bajo (GRADE 1C)

Los pólipos pediculados grandes tienen un riesgo mayor de hemorragia pospolipectomía (HPP) ${ }^{97}$; sin embargo, es posible tratarlos exitosamente hasta en el $90 \%$ de los casos, incluso cuando miden $>2 \mathrm{~cm}$ (90.3\%; IC 95\%: 88.2-92.5\%) ${ }^{138}$.

La colocación de endoloops, clips y la inyección submucosa de adrenalina han demostrado disminuir el riesgo de HPP en la mayoría de los casos, siendo más efectivos los dos primeros métodos mencionados ${ }^{139-144}$.

\section{LA RESECCIÓN ENDOSCÓPICA DE LA MUCOSA EN PÓLIPOS SÉSILES MAYORES A 2 CM LOGRA TASAS DE RESECCIÓN COMPLETA ACEPTABLES}

Recomendación fuerte. Nivel de evidencia moderado (GRADE 1B)

La mayoría de los pólipos mayores de $2 \mathrm{~cm}$, cuando se usa AC, suele extirparse en fragmentos (piecemeal). Esto incrementa el riesgo de recurrencia y dificulta una adecuada evaluación histológica. Para evitar estas limitaciones, se recomienda realizar resección completa en un bloque mediante REM.

Las ventajas de la REM son: es una técnica relativamente simple, requiere pocos instrumentos, tiene menos complicaciones, mayor disponibilidad y ha demostrado su utilidad en la mayoría de los pólipos grandes.

Intentar una resección completa se acompaña de mayor riesgo de lesiones térmicas y perforación. La principal desventaja de la REM es que la resección 
frecuentemente se realiza en fragmentos, lo que no permite una evaluación histopatológica precisa. Tiene una posibilidad de recurrencia del 15 al $20 \%$ que se puede tratar por endoscopia $97,138,145-147$.

El tamaño, la morfología, localización y experiencia del endoscopista son factores que determinan la posibilidad de resección en bloque de pólipos $>20 \mathrm{~mm}$, la cual se ha reportado ser del 16 al $48 \%$.

$\mathrm{Si}$ al intentar la REM, la inyección de la submucosa no logra una elevación adecuada, esto indica posible invasión neoplásica; esto en conjunto con otros signos morfológicos sugestivos de malignidad contraindican el procedimiento.

De acuerdo con un metaanálisis y guías europeas, la REM en pólipos grandes es efectiva y segura. En manos expertas logra una resección completa hasta en el 90 al $96 \%$ de los casos, con pocas complicaciones $1.5 \%$ de perforación, $6.5 \%$ de hemorragia, $0.08 \%$ de mortalidad y $0.8 \%$ necesidad de cirugía) $)^{97,138}$.

\section{LOS PACIENTES CON PÓLIPOS COMPLEJOS MAYORES A 20 MM DEBERÍAN SER REMITIDOS A CENTROS ESPECIALIZADOS PARA SU TRATAMIENTO MEDIANTE DISECCIÓN ENDOSCÓPICA DE LA SUBMUCOSA}

Recomendación fuerte. Nivel de evidencia moderada (GRADE 1B)

Los pacientes con pólipos sésiles grandes (> $20 \mathrm{~mm}$ ), difíciles, complejos, con crecimiento lateralmente extendido y sobre todo con posibilidad de malignidad deben referirse a centros especializados con endoscopistas expertos en técnicas como la disección endoscópica de la submucosa (DES) ${ }^{148,149}$.

En caso de sospecha de cáncer basado en las clasificaciones de París, Kudo y NICE (National Institute for Health and Care Excellence), está indicada la DES. El reporte de patología determinará la invasión a la submucosa.

Las ventajas de la DES son lograr extraer la lesión en una sola pieza y permitir una evaluación histopatológica precisa, pues facilita la identificación de los márgenes del corte. La tasa de recurrencia comparada con la REM es baja (DES: $1 \%$ vs. REM: $15-20 \%$ ) y logra la curación de algunos casos de cáncer temprano. En caso de extensión a capas profundas estos pacientes deben ser referidos a cirugía ${ }^{150-152}$.

Las desventajas de la DES son el riesgo de perforación (DES: 2-14\% vs. REM: 0.4-1.3\%), se considera una técnica compleja que requiere capacitación avanzada, por lo que generalmente se realiza en pocos centros $^{149}$.
Está en desarrollo el primer estudio comparativo aleatorizado de REM vs. DES en pólipos grandes no pediculados que proveerá de información relacionada con efectividad, seguridad, calidad de vida y costo-efectividad de ambas técnicas ${ }^{152}$.

\section{Prevención de complicaciones}

La complicaciones relacionadas con la colonoscopia forman parte de los parámetros de calidad. La complicación más frecuente de la colonoscopia de escrutinio es la HPP, que se presenta entre el 0.3 y el $1.1 \%$ de los casos. La HPP puede ser temprana cuando se presenta en las primeras 24 horas del procedimiento y tardía cuando se presenta 24 horas y hasta 14 días después del procedimiento ${ }^{152}$.

\section{EXISTEN FACTORES DE RIESGO QUE PREDICEN EL DESARROLLO DE HEMORRAGIA POSPOLIPECTOMÍA}

Recomendación fuerte. Nivel de evidencia alto (GRADE 1A)

Los factores que predicen HPP son aquellos relacionados con el pólipo (localización y características del pólipo), el procedimiento (técnica de resección empleada) y el paciente (edad mayor a 65 años, enfermedades cardiovasculares, renales, uso de antiagregantes y anticoagulantes).

Los pólipos $\geq 10 \mathrm{~mm}$ tienen 2 a 4.5 veces mayor riesgo de HPP y este riesgo se incrementa conforme incrementa el tamaño del pólipo. Aquellos pólipos localizados en el colon derecho tienen 2.6 a 4.6 veces mayor riesgo de HPP. Los pólipos pediculados con tallo grueso o lesiones lateralmente extendidas, y adenomas 0 adenocarcinomas se asocian también con mayor riesgo ${ }^{153}$.

La edad > 65 años (RM: 1.37; IC 95\%: 1.02-1.83), comorbilidades cardiovasculares (RM: 2.08; IC 95\%: 1.45-2.99), insuficiencia renal crónica (RM: 3.29 ; IC 95\%: 1.84-5.87), uso de anticoagulantes (RM: 3.71 ; IC 95\%: 1.05-13.05), pólipos > $1 \mathrm{~cm}$ (RM: 2.38; IC 95\%: 1.78-3.18), pólipos pediculados o lateralmente extendidos (RM: 1.42; IC 95\%: 1.06-1.89), una preparación colónica subóptima (RM: 1.54; IC 95\%: 1.09-2.19), uso de corriente de corte (RM: 6.95; IC 95\%: 4.42-10.94) y polipectomía fría inadvertida (RM: 7.15; IC 95\%: 3.1316.36) se asocian con HPP temprana ${ }^{154}$.

Los factores de riesgo para HPP tardía han sido poco estudiados; el tamaño del pólipo ha sido identificado como uno de los pocos factores independientes. 
Pólipos $\geq 10 \mathrm{~mm}$ tienen 4.5 veces más riesgo (IC 95\%: 2.0-10.3; $p=0.0003$ ) de HPP tardía.

La hipertensión arterial se asocia a una RM ajustada de 5.6 (IC 95\%: 1.8-17.2; $p=0.001$ ), mientras que la localización del pólipo y el método de resección no parecen afectar el riesgo de sangrado ${ }^{155}$.

Es importante documentar el uso de antiagregantes, anticoagulantes y cuándo reiniciarlos. Pacientes en tratamiento con antitrombóticos presentan HPP hasta en un $1.19 \%$ (IC 95\%: 0.91-1.54\%) de las veces, aquellos con anticoagulantes orales (p. ej., warfarina) en un $0.66 \%$, con clopidogrel en un $0.84 \%$ y con aspirina en un $0.92 \%$. La terapia puente se asocia a HPP hasta en el $15 \%$ de los casos. Estos riesgos parecen relacionarse directamente con el tamaño del pólipo, pues aquellos que sangran suelen ser mayores de $10 \mathrm{~mm}$.

El reinicio temprano de terapia antitrombótica (1 semana posterior a la polipectomía) comparado con controles incrementa la tasa y riesgo de HPP tardía (75 vs. $34 \%$; RM: $4.50 ; p<0.001$ ). El uso de terapia puente también incrementa la frecuencia de sangrado comparado con pacientes sin tratamiento (76 vs. $18 \%$; RM: 14.4; $p=0.001$ ) respectivamente. El manejo de estos pacientes debe hacerse en conjunto con hematología ${ }^{156}$.

El manejo endoscópico y no endoscópico (radiología intervencionista y quirúrgico) de la HPP deberá individualizarse. Recomendaciones al respecto se describen en la guía de hemorragia de tubo digestivo bajo publicada por la AMEG ${ }^{143,157-159}$.

Otras complicaciones que se deben tener en cuenta para establecer un diagnóstico y resolución oportunas son la perforación colónica y el síndrome pospolipectomía. Esta puede ocurrir en el 0.8 al $2.1 \%$ de polipectomías y entre el 2 y el 14\% de DES ${ }^{160,161 .}$

El sitio de perforación más frecuentemente es el sigmoides, especialmente en pacientes con enfermedad inflamatoria activa, cirugías abdominales previas, cirrosis hepática e ingesta crónica de esteroides ${ }^{162}$. El tratamiento puede ser endoscópico o quirúrgico ${ }^{97,161,162}$. El síndrome de pospolipectomía es poco frecuente, su incidencia se estima entre el 0.5 y el $1.2 \%$ de todas las polipectomías (hasta un $4.8 \%$ posterior a DES). Ocurre secundario al daño térmico generado por el AC que afecta mucosa, muscularis propria y serosa (lesión térmica transmural) sin que ocurra una perforación ${ }^{163,164}$. Se manifiesta por dolor abdominal, fiebre, leucocitosis y datos de irritación peritoneal en las 12 horas 05 días después del procedimiento ${ }^{165}$. El manejo es conservador, a base de hidratación y antibióticos intravenosos ${ }^{161}$.

\section{Conclusiones}

La presente guía de la AMEG sobre calidad en coIonoscopia es el resultado de un análisis multidisciplinario basado en la evidencia disponible hasta el momento de su elaboración y en la opinión de especialistas expertos. Esta guía tiene la intención de proveer recomendaciones que ayuden a mejorar y mantener la calidad de una colonoscopia.

\section{Agradecimientos}

Agradecemos a Laboratorio Asofarma por su apoyo para llevar a cabo la reunión final de consenso.

\section{Financiamiento}

Laboratorios Asofarma proporcionó la financiación económica para la reunión final de los autores del consenso.

\section{Conflicto de intereses}

Los participantes declaran no tener algún conflicto de interés relacionado con esta guía.

\section{Responsabilidades éticas}

Protección de personas y animales. Los autores declaran que para esta investigación no se han realizado experimentos en seres humanos ni en animales.

Confidencialidad de los datos. Los autores declaran que en este artículo no aparecen datos de pacientes.

Derecho a la privacidad y consentimiento informado. Los autores declaran que en este artículo no aparecen datos de pacientes.

\section{Bibliografía}

1. Fink A, Kosecoff J, Chassin M, Brook RH. Consensus methods: characteristics and guidelines for use. Am J Public Health. 1984;74:979-83.

2. Grading tutorial. Grading recommendations in UpToDate ${ }^{\circledR}$ [Internet]. Wolters Kluwer [consultado: 26 de junio 2019]. Disponible en: http://www. uptodate.com/home/grading-tutorial

3. Guyatt GH, Oxman AD, Vist GE, Kunz R, Falck-Ytter Y, Alonso-Coello P, et al.; GRADE Working Group. GRADE: an emerging consensus on rating quality of evidence and strength of recommendations. BMJ. 2008;336:924-6.

4. Jang JY, Chun HJ. Bowel preparation as quality indicator for colonoscopy. World J Gastroenterol. 2014;20:2746-50.

5. Rex DK, Schoenfeld PS, Cohen J, Pike IM, Adler DG, Fennerty MB, et al. Quality indicators for colonoscopy. Gastrointest Endosc. 2015;81(1):31-53.

6. Froehlich F, Wietlisbach V, Gonvers JJ, Burnand B, Vader JP. Impact of colonic cleansing on quality and diagnostic yield of colonoscopy: the European Panel of Appropriateness of Gastrointestinal Endoscopy European multicenter study. Gastrointest Endosc. 2005;61(3):378-84. 
7. Lebwohl B, Neugut Al. Post-colonoscopy recommendations after inadequate bowel preparation: all in the timing. Dig Dis Sci. 2013;58;2135-7.

8. Lebwohl B, Kastrinos F, Glick M, Rosenbaum AJ, Wang T, Neugut Al. The impact of suboptimal bowel preparation on adenoma miss rates and the factors associated with early repeat colonoscopy. Gastrointest Endosc. 2011;73:1207-14

9. Bowles CJ, Leicester R, Romaya C, Swarbrick E, Williams CB, Epstein O. A prospective study in colonoscopy practice in the UK today: are we adequately prepared for national colorectal cancer screening tomorrow? Gut. 2014;53:277-83.

10. Nelson DB, McQuaid KR, Bond JH, Lieberman DA, Weiss DG, Johnston TK. Procedural success and complications of large-scale screening colonoscopy. Gastrointest Endosc. 2002;55:307-14.

11. ASGE Standards of Practice Committee, Saltzman JR, Cash BD, Pasha SF, Early DS, Muthusamy VR, Khashab MA, et al. Bowel preparation before colonoscopy. Gastrointest Endosc. 2015;81(4):781-94.

12. Rembacken B, Hassan C, Riemann JF, Chilton A, Rutter M, Dumonceau JM, et al. Quality in screening colonoscopy: position statement of the European Society of Gastrointestinal Endoscopy (ESGE). Endoscopy. 2012;44:957-68.

13. Kastenberg D, Bertiger G, Brogadir S. Bowel preparation quality scales for colonoscopy. World J Gastroenterol. 2018;24:2833-43.

14. Kim EJ, Park YI, Kim YS, Park WW, Kwon SO, Parl KS, et al. A Korean experience of the use of Boston bowel preparation scale: A valid and reliable instrument for colonoscopy-oriented research. Saudi J Gastro. 2016;20(4):219-24.

15. Parmar R, Martel M, Rostom A, Barkun AN. Validated scales for colon cleansing: a systematic review. Am J Gastroenterol. 2016;111:197-204.

16. Lorenzo-Zúñiga $\mathrm{V}$, Moreno de Vega V, Boix J. Preparación para colonoscopia: tipos de productos y escalas. Rev Esp Enferm Dig. 2012; 104:426-31.

17. Meneses S, Kim HM, Elliott EE, Mickevicius JL, Graustein BB, Schoenfeld PS. The impact of fair colonoscopy preparation on colonoscopy use and adenoma miss rates in patients undergoing outpatient colonoscopy. Gastrointest Endosc. 2013:78:510-16.

18. Calderwood A, Schroy PC 3rd, Lieberman DA, Logan JR, Zurfluh M Jacobson BC. Boston Bowel Preparation Scale scores provide a standardized definition of adequate for describing bowel cleanliness. Gastrointest Endosc. 2014;80:269-76.

19. Chang CW, Shih SC, Wang HY, Chu CH, Wang TE, Hung CY, et al. Meta-analysis: The effect of patient education on bowel preparation for colonoscopy. Endosc Int Open. 2015;3(6):E646-E652.

20. Gkolfakis P, Tziatzios G, Papanikolaou IS, Triantafyllou K. Strategies to improve inpatients' quality of bowel preparation for colonoscopy: A systematic review and meta-analysis. Gastroenterol Res Pract. 2019;2019:5147208

21. Park JS, Kim MS, Kim H, Kim SI, Shin $\mathrm{CH}$, Lee $\mathrm{HJ}$, et al. A randomized controlled trial of an educational video to improve quality of bowel preparation for colonoscopy. BMC Gastroenterol. 2016;16(1):64.

22. Elvas L, Brito D, Areia M, Carvalho R, Alves S, Saraiva S, et al. Impact of personalised patient education on bowel preparation for colonoscopy: Prospective randomised controlled trial. GE Port J Gastroenterol. 2017;24(1):22-30.

23. Guo X, Yang Z, Zhao L, Leung F, Luo H, Kang X, et al. Enhanced instructions improve the quality of bowel preparation for colonoscopy: a meta-analysis of randomized controlled trials. Gastrointest Endosc. 2017;85(1):90-7.

24. Allencherril R, Al-Saadi Y, Kuntz DM. Knowledge of bowel preparation for inpatient colonoscopy: A baseline quality improvement study of nursind staff at a tertiary care hospital. Gastrointest Endosc. 2018; 87(6S):AB527.

25. Lee YJ, Kim ES, Park KS, Cho KB, Jang BK, Chung WJ, et al. Education of ward nurses influences the quality of inpatient's bowel preparation for colonoscopy. Medicine (Baltimore) 2015; 94(34): e1423.

26. Song GM, Tian $X, M a L$, et al. Regime for bowel preparation in patients scheduled to colonoscopy: Low-residue diet or clear liquid diet? Evidence from systematic review with power analysis. Medicine (Baltimore) 2016;95(1):e2432.

27. Nguyen DL, Jamal MM, Nguyen ET, et al. Low-residue versus clear liquid diet before colonoscopy: a meta-analysis of randomized controlled trials. Gastrointest Endosc. 2016;83(3):499-507.

28. Gimeno-García AZ, de la Barreda Hauser R, Reygosa C, et al. Impact of a 1-day versus 3-day low residue diet on bowel cleansing quality before colonoscopy: a randomised controlled trial. Endoscopy. 2019; 51(7):628-36.

29. Murcio-Pérez E, Tellez-Ávila F. Opciones de preparación para colonoscopia. Endoscopia. 2012:24:23-31.

30. Bechtold ML, Mir F, Puli SR, et al. Optimizing bowel preparation for colonoscopy: a guide to enhance quality of visualization. Ann Gastroenterol. 2016;29:137-46.

31. Parekh PJ, Oldfield EC, Johnson DA. Bowel preparation for colonoscopy: what is best and necessary for quality. Current Opin Gastroenterol. 2019;35:51-7
32. Enestvedt BK, Tofani C, Laine LA, et al. 4-Liter split-dose polyethylene glycol is superior to other bowel preparations, based on systematic review and meta-analysis. Clin Gastroenterol Hepatol. 2012;10:1225-31.

33. Clark RE, Godfrey JD, Choudhary A, et al. Low-volume polyethylene glycol and bisacodyl for bowel preparation prior to colonoscopy: a meta-analysis. Gastroenterol. 2013;26(4):319-24.

34. Wang X, Coa G, Moa W. Comparison of two-liter polyethylene glycol plus bisacodyl versus four-liter polyethylene glycol as preparation for colonoscopy in colon disease: a systemic review and meta-analysis. Int J Clin Exp Med. 2018;11:5418-29.

35. Macías Ángeles YR, Saraiba Reyes M, Tejada García RA, et al. Comparación de la efectividad de 2 esquemas de preparación intestinal para colonoscopia en el Hospital General de México. Endoscopia. 2015;27:98-103.

36. Jin Z, Lu Y, Gong B. Systematic review and meta-analysis: sodium picosulfate/magnesium citrate vs. polyethylene glycol for colonoscopy preparation. Eur J Clin Pharmacol. 2016;2:523-32.

37. Curran MP, Plosker GL. Oral sodium phosphate solution: A review of its use as a colonic cleanser. Drugs. 2004;64:1697-714.

38. Tan JJ, Tjandra JJ. Which is the optimal bowel preparation for colonoscopy: a meta-analysis. Colorectal Dis. 2006;8:247-58.

39. Mamula P, Adler DG, Conway JD, et al. Colonoscopy preparation. Gastroint Endosc. 2009;69(7):1201-09.

40. Johnson DA, Barkun AN, Cohen LB, et al. Optimizing adequacy of bowel cleansing for colonoscopy: recommendations from the US Multi-Society Task Force on Colorectal Cancer. Am J Gastroenterol. 2014:109(10):1528-45.

41. Kilgore TW, Abdillahi AA, Szary NM, et al. Bowel preparation with a split-dose polyethylene glycol before colonoscopy: a meta-analysis of randomized controlled trials. Gastrointest Endosc. 2011;73:1240-5.

42. Martel M, Barkun AN, Menard C, et al. Split-dose preparations are superior to day-before bowel cleansing regimens: A meta-analysis. Gastroenterology. 2015;149:79-88.

43. Bucci C, Rotondano G, Hassan C, et al. Optimal bowel cleansing for colonoscopy: Split the dose! A series of meta-analyses of controlled studies. Gastrointest Endosc. 2014;80:566-76.

44. Bucci $C$, Zingone $F$, Schettino $P$, et al. Same-day regimen as an alternative to split preparation for colonoscopy: A systematic review with meta-analysis. Gastroenterol Res Pract. 2019;2019:7476023.

45. Hassan C, Bretthauer M, Kaminski MF, et al. Bowel preparation for colonoscopy: European Society of Gastrointestinal Endoscopy (ESGE) Guideline. Endoscopy. 2013;45:142-50.

46. Siddiqui AA, Yang K, Spechler SJ et al. Duration of the interval between the completion of bowel preparation and the start of colonoscopy predicts bowel-preparation quality. Gastrointest Endosc. 2009;69:700-6.

47. Huffman M, Unger RZ, Thatikonda C, et al. Split-dose bowel preparation for colonoscopy and residual gastric fluid volume: an observational study. Gastrointest Endosc. 2010;72:516-22.

48. Wexner SD, Beck DE, Baron TH, et al. A consensus document on bowel preparation before colonoscopy: prepared by task force from the American Society of Colon and Rectal Surgeons (ASCRS), the American Society for Gastrointestinal Endoscopy (ASGE), and the Society of American Gastrointestinal and Endoscopic Surgeons (SAGES). Surg Endosc. 2006;20:1147-60.

49. Martinek J, Hess J, Delarive J, et al. Cisapride does not improve precoIonoscopy bowel preparation with either sodium phosphate or polyethylene glycol electrolyte lavage. Gastrointest Endosc. 2001;54:180-5.

50. Katsinelos P, Pilpilidis I, Paroutoglou G, et al. The administration of cisapride as an adjuvant to PEG-electrolyte solution for colonic cleansing: a double-blind randomized study. Hepatogastroenterology. 2005;52:441-43.

51. Sanaka MR, Super DM, Mullen KD et al. Use of tegaserod along with polyethylene glycol electrolyte solution for colonoscopy bowel preparation: a prospective, randomized, double-blind, placebo-controlled study. Aliment Pharmacol Ther. 2006;23:669-74.

52. Mishima $Y$, Amano $Y$, Okita K, et al. Efficacy of prokinetic agents in improving bowel preparation for colonoscopy. Digestion. 2008;77:166-72.

53. Ruiz-Romero D, Téllez-Ávila FI. Preparación para colonoscopia en 2016: recomendaciones actuales utilizando datos nacionales. Endoscopia. 2016;28:81-9.

54. Parra-Blanco A, Ruiz A, Álvarez-Lobos $M$, et al. Achieving the best preparation for colonoscopy. World J Gastroenterol. 2014;21:17709-26.

55. Sharara Al, Abou Mrad RR. The modern bowel preparation in colonoscopy. Gastroenterol Clin North Am. 2013:42:577-98.

56. Baxter N, Sutradhar R, Forbes DD, et al. Analysis of administrative data finds endoscopist quality measures associated with post-colonoscopy colorectal cancer. Gastroenterology. 2011;140:65-72.

57. Williams C. Insertion technique. En: Waye JD, Rex DK, Williams CB, editores. Colonoscopy principles and practice. 2nd ed. Londres: Wiley-Blackwell; 2009. pp. 537-559.

58. Rex DK, Chen SC, Overhiser AJ. Colonoscopy technique in consecutive patients referred for prior incomplete colonoscopy. Clin Gastroenterol Hepatol. 2007;5:879-83.

59. Rex DK. Still photography versus videotaping for documentation of cecal intubation: a prospective study. Gastrointest Endosc. 2000;51:451-9.

60. Marshall JB, Barthel JS. The frequency of total colonoscopy and terminal ileal intubation in the 1990s. Gastrointest Endosc. 1993;39:518-20. 
61. Training Committee 2010-2011, Sedlack RE, Shami VM, et al. Colonoscopy core curriculum. Gastrointest Endosc. 2012;76:482-90.

62. Hoff G, Volker M, Bretthauer M, et al. Gastronet survey on the use of one or two-person technique for colonoscopy insertion. BMC Gastroenterol. 2011:11:73.

63. Hsu CM, Lin WP, Su MY, et al. Factors that influence cecal intubation rate during colonoscopy in deeply sedated patients. J Gastroenterol Hepatol. 2012;27:76-80.

64. Paggi S, Rondonotti E, Amato A, et al. One or two operator technique and quality performance of colonoscopy: A randomised controlled trial. Dig Liver Dis. 2014;46(7):616-20.

65. Xu X, Ni D, Lu Y, et al. Diagnostic application of water exchange colonoscopy: A meta-analysis of randomized controlled trials. Int Med Res. 2019;47:515-27

66. Shi $X$, Tian $D, Y e X$, et al. Is water exchange superior to water immersion in detecting adenomas during colonoscopies? Results from a Bayesian network meta-analysis. Oncotarget. 2018;9(55):30679-93

67. Cadoni S, Falt P, Rondonotti E, et al. Water exchange for screening colonoscopy increases adenoma detection rate: a multicenter, double-blinded, randomized controlled trial. Endoscopy. 2017;49(5):456-67.

68. Cadoni S, Leung FF. Water-assisted colonoscopy. Curr Treat Options Gastroenterol. 2017:15:135-54

69. Brooker JC, Saunders BP, Shah SG, et al. A new variable stiffness colonoscope makes colonoscopy easier: a randomised controlled trial. Gut. 2000:46:801-5.

70. Othman MO, Bradley AG, Roy PK. Is variable stiffness colonoscope better than regular adult colonoscope for colonoscopy? Meta-analysis of randomized controlled trials. Gastrointest Endosc. 2006;63(5):AB98.

71. Lee DW, Li AC, Ko CW, et al. Use of a variable-stiffness colonoscope decreases the dose of patient-controlled sedation during colonoscopy: a randomized comparison of 3 colonoscopes. Gastrointest Endosc. 2007:65(3):424-9.

72. Othman Mo, Bradley AG, Choudhary A, et al. Variable stiffness colonoscope versus regular adult colonoscope: meta-analysis of randomized controlled trials. Endoscopy. 2009;41(1):17-24

73. ASGE Technology Committee, Trindale AJ, Lichtenstein DR, et al. Devices and methods to improve colonoscopy completion (with videos). Gastrointest Endosc. 2018;87(3):625-34.

74. Morgan J, Thomas K, Lee-Rochibaud H, et al. Transparent cap colonoscopy versus standard colonoscopy to improve caecal intubation. Cochrane Database Syst Rev. 2012;12:CD008211.

75. Bretthauer M, Hoff G, Thiis-Evensen E, et al. How much gas do we insufflate during colonoscopy? Air and carbon dioxide volumes insufflated during colonoscopy. Gastrointest Endosc. 2003;58:203-6.

76. Stevenson GW, Wilson JA, Wilkinson J. Pain following colonoscopy: elimination with carbon dioxide. Gastrointest Endosc. 1992;38:564-7.

77. Bretthauer M, Thiis-Evensen E, Huppertz-Hauss G, et al. NORCCAP (Norwegian Colorectal Cancer Prevention): a randomized trial to assess the safety and efficacy of carbon dioxide versus air insufflation in colonoscopy. Gut. 2002:50:604-7.

78. Sumanac K, Zealley I, Fox B. et al. Minimizing postcolonoscopy abdominal pain by using $\mathrm{CO} 2$ insufflation: a prospective, randomized, double blind, controlled trial evaluating a new commercially available $\mathrm{CO} 2$ delivery system. Gastrointest Endosc. 2002;56:190-4.

79. Price HL. Effects of carbon dioxide on the cardiovascular system. Anesthesiology. 1960;21:652-63.

80. Church J, Delaney C. Randomized controlled trial of carbon dioxide insufflation during colonoscopy. Dis Colon Rectum. 2003;46:322-26.

81. Bretthauer M, Lynge AB, Thiis-Evensen E, et al. Carbon dioxide insufflation in colonoscopy: safe and effective in sedated patients. Endoscopy. 2005:37:706-9.

82. Wu J, Hu B. The role of carbon dioxide insufflation in colonoscopy: a systematic review and meta-analysis. Endoscopy. 2012;44:128-36.

83. Rogers BH. The safety of carbon dioxide insufflation during colonoscopic electrosurgical polypectomy. Gastrointest Endosc. 1974;20:115-17.

84. Bretthauer M, Hoff G. The use of $\mathrm{CO} 2$ in colonoscopy. Gastrointest Endosc. 2003:57:436-8.

85. Zhao SB, Yang X, Fang J. Effect of left lateral tilt-down position on cecal intubation: a 2-center, pragmatic, randomized controlled trial. Gastrointest Endosc. 2018;87(3):852-61.

86. Vergis N, McGrath AK, Stoddart CH, et al. Right or Left in Colonoscopy (ROLCOL)? A randomized controlled trial of right-versus left sided start. Am J Gastroenterol. 2015;110(11):1576-81.

87. Pfau PR. Colonoscopy and kinematics: what is your path length and tip angulation? Gastrointest Endosc. 2011;73(2):322-4.

88. Obstein KL, Patil VD, Jayender J, et al. Evaluation of colonoscopy technical skill levels by use of an objective kinematic-based system. Gastrointest Endosc. 2011;73:315-21.

89. Shah SG, Saunders BP, Brooker JC, et al. Magnetic imaging of colonoscopy: an audit of looping, accuracy and ancillary maneuvers. Gastrointest Endosc. 2000:52(1):1-8.

90. Kamal A, van Dam J. Reduced loop formation using a computer-assisted colonoscope (the NeoGuide system) compared with standard colonoscopy. Gastrointest Endosc. 2006;63(5):AB228.
91. Coderre S, Anderson J, Rikers R, et al. Early use of magnetic endoscopic imaging by novice colonoscopists: improved performance without increase in workload. Can J Gastroenterol. 2010;24(12):727.

92. Rex DK, Boland CR, Dominitz JA, et al Colorectal cancer screening: recommendations for physicians and patients from the US multi-society task force on colorectal cancer. Gastrointest Endosc. 2017;86(1):18-33.

93. Rex DK, Petrini JL, Baron TH, et al. Quality indicators for colonoscopy. Gastrointest Endosc. 2006;63(4 Suppl):S16-28.

94. Aarons CB, Shanmugan S, Bleier JIS. Management of malignant colon polyps: Current status and controversies. World J Gastroenterol. 2014:20(43):16178-83

95. Sánchez del Río A, Pérez Romero S, López-Picazo S, et al. Indicadores de calidad en colonoscopia. Rev Esp Enferm Dig. 2018;110:316-26.

96. Ferlitsch M, Moss A, Hassan C, et al. Colorectal polypectomy and endoscopic mucosal resection (EMR): European Society of Gastrointestinal Endoscopy (ESGE) Clinical Guideline. Endoscopy. 2017;49(3):270-97.

97. Bujanda L, Cosme A, Gil I, et al. Malignant colorectal polyps. World J Gastroenterol. 2010;16:3103-11.

98. Leung FW, Amato A, Ell C, et al. Water-aided colonoscopy: a systematic review. Gastrointest Endosc. 2012;76(3):657-6.

99. Leung FW, Aharonian HS, Leung JW, et al. Impact of a novel water method on scheduled unsedated colonoscopy in U.S. veterans. Gastrointest Endosc. 2009;69(3):546-50.

100. Leung JW, Do LD, Siao-Salera RM, et al. Retrospective analysis showing the water method increased adenoma detection rate - a hypothesis generating observation. J Interv Gastroenterol. 2011;1(1):3-7.

101. Ramirez FC, Leung FW. A head-to-head comparison of the water vs. air method in patients undergoing screening colonoscopy. J Interv Gastroenterol. 2011;1(3):130-5

102. Takano N, Yamaji $\mathrm{Y}$, Kajiwara $\mathrm{H}$, et al. A randomized controlled trial of the usefulness of cap-fitted colonoscopy on the polyp detection. Gastrointest Endosc. 2008:67(5):AB303

103. Facciorusso A, Del Prete V, Buccino RV, et al. Comparative efficacy of colonoscope distal attachment devices in increasing rates of adenoma detection: a network meta-analysis. Clin Gastroenterol Hepatol. 2018;16(8):1209-19.

104. Triantafyllou K, Gkolfakis P, Tziatzios G, et al. Effect of endocuff use on colonoscopy outcomes. A systemantic review y meta-analysis. World J Gastroenterol. 2019;25(9):1158-70.

105. Williet N, Tournier Q, Vernet C, et al. Effect of endocuff-assisted colonoscopy on adenoma detection rate: meta-analysis of randomized controlled trials. Endoscopy. 2018;50(9):846-60.

106. Desai M, Bilal M, Hamade N, et al. Increasing adenoma detection rates in the right side of the colon comparing retroflexion with a second forward view: a systematic review. Gastrointest Endosc. 2018:89(3):453-9.

107. Ngu WS, Rees C. Can technology increase adenoma detection rate? Therap Adv Gastroenterol. 2018;11:1756283X17746311.

108. Sulz MC, Kröger A, Prakash M, et al. Meta-analysis of the effect of bowel preparation on adenoma detection: Early adenomas affected stronger than advanced adenomas. PLoS One. 2016;11(6) e0154149.

109. Clark BT, Rustagi $T$, Laine $L$. What level of bowel prep quality requires early repeat colonoscopy: systematic review and meta-analysis of the impact of preparation quality on adenoma detection rate. Am J Gastroenterol. 2014;109(11):1714-24.

110. Radaelli F, Meucci G, Sgroi G, et al. Technical performance of colonoscopy: the key role of sedation/analgesia and other quality indicators. Am J Gastroenterol. 2008;103:1122-30.

111. ASGE Standards of Practice Committee, Early DS, Lightdale JR, et al. Guidelines for sedation and anesthesia in Gl endoscopy. Gastrointest Endosc. 2018:87(2):327-37.

112. Wang A, Hoda KM, Holub JL, et al. Does level of sedation impact detection of advanced neoplasia? Dig Dis Sci. 2010;55(8):2337-43

113. Nakshabendi R, Berry AC, Munoz JC, et al. Choice of sedation and its impact on adenoma detection rate in screening colonoscopies. Ann Gastroenterol. 2016;29(1):50-5

114. Turse E, Dailey FE, Bechtold ML. Impact of moderate versus deep sedation on adenoma detection rate in index average-risk screening colonoscopies. Gastrointest Endosc. 2019;90(3):502-5.

115. Paspatis GA, Tribonias G, Manolaraki MM, et al. Deep sedation compared with moderate sedation in polyp detection during colonoscopy: a randomized controlled trial. Colorectal Dis. 2011;13(6):e137-e144.

116. Heitman SJ, Ronksley PE, Hilsden RJ, et al. Prevalence of adenomas and colorectal cancer in average risk individuals: A systematic review and meta-analysis. Clin Gastroenterol Hepatol. 2009:7(12):1272-8.

117. van Rjin JC, Reitsma JB, Stoker J, et al. Polyp miss rate determined by tandem colonoscopy: a systematic review. Am J Gastroenterol. 2006;101:343-50.

118. Hoffman A, Sar F, Goetz M, et al. High definition colonoscopy combined with i-Scan is superior in the detection of colorectal neoplasias compared to standard video colonoscopy. Endoscopy. 2010:42:827-33.

119. Subramanian V, Mannath J, Hawkey C, et al. High definition colonoscopy vs standard video endoscopy for the detection of colonic polyps: a meta-analysis. Endoscopy. 2011;43:499-505. 
120. Brown SR, Baraza W. Chromoscopy versus conventional endoscopy for the detection of polyps in the colon and rectum. Cochrane Database Sys Rev. 2010;(10):CD006439.

121. Kaminski MF, Hassan $C$, Bisschops $R$, et al. Advanced imaging for detection and differentiation of colorectal neoplasia: European Society of Gastrointestinal Endoscopy (ESGE) Guideline. Endoscopy. 2014;46(5):435-49.

122. Dik VK, Moons LM, Siersema PD. Endoscopic innovations to increase the adenoma detection rate during colonoscopy. World J Gastroenterol. 2014;20(9):2200-11.

123. Khakimov N, Khasanova G, Ershova K, et al. Screening for colon cancer: A test for occult blood. Int J of Risk Saf Med. 2015-27(Supp 1):S110-S111.

124. Wong JCT, Chiu HM, KimHS, et al. Adenoma detection rates in colonoscopies for positive fecal immunochemical tests versus direct screening colonoscopies. Gastrointest Endosc. 2019;89(3):607-13.

125. Imperiale TF, Ransohoff DF, Itzkowitz SH. Multitarget stool DNA testing for colorectal-cancer screening. N Engl J Med. 2014;371(2):187-88.

126. Lee CK, Shim JJ, Jang JY. Cold snare polypectomy vs cold forceps polypectomy using double-biopsy technique for removal of diminutive colorectal polyps: a prospective randomized study. Am J Gastroenterol. 2013;108:1593-600.

127. Kim JS, Lee BI, Choi H, et al. Cold snare polypectomy versus cold forceps polypectomy for diminutive and small colorectal polyps: a randomized controlled trial. Gastrointest Endosc. 2015;81:741-7.

128. Komeda Y, Kashida H, Sakurai T, et al. Removal of diminutive colorectal polyps: a prospective randomized clinical trial between cold snare polypectomy and hot forceps biopsy. World J Gastroenterol. 2017;23:328-35.

129. Kawamuna T, Takeuchi $Y$, Asai S, et al. A comparison of the resection rate for cold and hot snare polypectomy for 4-9 mm colorectal polyps: a multicentre randomized controlled trial (CRESCENT study). Gut. 2018:67:1950-7.

130. Shinozaki S, Kobayashi Y, Hayashi Y, et al. Efficacy and safety of cold versus snare polypectomy for resecting small colorectal polyps: systematic review and meta-analysis. Digest Endosc. 2018;30:592-9.

131. Yung YS, Park CH, Nam E, et al. Comparative efficacy of cold polypectomy techniques for diminutive colorectal polyps: a systematic review and network meta-analysis. Surg Endosc. 2018;32:1149-59.

132. Horiuchi A, Ikuse T, Tanaka N. Cold snare polypectomy: indications, devices, techniques, outcomes and future. Dig Endosc. 2019;31:372-7.

133. Kuwai $T$, Yamada $T$, Toyokawa $T$, et al. Local recurrence of diminutive polyps after cold forceps polypectomy with jumbo forceps followed by magnified narrow-band imaging: a multicenter prospective study. Endoscopy. 2019;51:253-60.

134. Huh CW, Kim JS, Choi HH, et al. Jumbo biopsy forceps versus cold snares for removing diminutive colorectal polyps: a prospective randomized controlled trial. Gastrointest Endosc. 2019;90:105-11.

135. Pohl H, Srivastava A, Bensen SP, et al. Incomplete polyp resection during colonoscopy-results of the complete adenoma resection (CARE) study. Gastroenterology. 2013;144:417-23.

136. Kim HS, Jung HY, Park HJ, et al. Hot snare polypectomy with or without saline solution/epinephrine lift for the complete resection of small colorectal polyps. Gastrointest Endosc. 2018;87:1539-47.

137. Thoguluva Chandrasekar V, Spadaccini M, Aziz M, et al. Cold snare endoscopic resection of nonpedunculated colorectal polyps larger than $10 \mathrm{~mm}$ : a systematic review and pooled-analysis. Gastrointest Endosc. 2019;89:929-36.

138. Hassan C, Repici A, Sharma P, et al. Efficacy and safety of endoscopic resection of large colorectal polyps: a systematic review and meta-analysis. Gut. 2016:65:806-20.

139. Matsumoto $\mathrm{M}$, Kato $\mathrm{M}, \mathrm{Oba} \mathrm{K}$, et al. Multicenter randomized controlled study to assess the effect of prophylactic clipping on post-polypectomy delayed bleeding. Dig Endosc. 2016;5:570-6.

140. di Giorgio P, de Luca L, Calcagno G, et al. Detachable snare versus epinephrine injection in the prevention of postpolypectomy bleeding: a randomized and controlled study. Endoscopy. 2004;36:860-3.

141. Dobrowolski S, Dobosz M, Babicki A, et al. Prophylactic submucosal saline-adrenaline injection in colonoscopic polypectomy: prospective randomized study. Surg Endosc. 2004;18:990-3.

142. Paspatis GA, Paraskeva K, Theodoropoulou A, et al. A prospective, randomized comparison of adrenaline injection in combination with detachable snare versus adrenaline injection alone in the prevention of postpolypectomy bleeding in large colonic polyps. Am J Gastroenterol. 2006;101:2805-913.
143. Kouklakis G, Mpoumponaris A, Gatopoulou A, et al. Endoscopic resection of large pedunculated colonic polyps and risk of postpolypectomy bleeding with adrenaline injection versus endoloop and hemoclip: a prospective, randomized study. Surg Endosc. 2009;23:2732-7.

144. Park $\mathrm{CH}$, Jung $\mathrm{YS}$, Nam $\mathrm{E}$, et al. Comparison of efficacy of prophylactic endoscopic therapies for postpolypectomy bleeding in the colorectum: A systematic review and network meta-analysis. Am J Gastroenterol. 2016;111:1230-43

145. Draganov PV. Endoscopic mucosal resection vs endoscopic submucosal dissection for colon polyps. Gastroenterol Hepatol (N Y). 2018;14:50-2.

146. Rutter M, Chattree A, Barbour J, et al. British Society of Gastroenterology/Association of Coloproctologists of Great Britain and Ireland guidelines for the management of large non-pedunculated colorectal polyps. Gut. 2015;64:1847-73.

147. Herszényi L. The "difficult" colorectal polyps and adenomas: Practical aspects. Dig Dis. 2019;37:394-9.

148. Von Renteln D, Bouin M, Barkun AN. Current standards and new developments of colorectal polyp management and resection techniques. Expert Rev Gastroenterol Hepatol. 2017;11:835-42.

149. Draganov PV, Wang AY, Othman MO, et al. AGA Institute Clinical Practice Update: Endoscopic submucosal dissection in the United States. Clin Gastroenterol Hepatol. 2019;17:16-25.

150. Fuccio L, Ponchon T. Colorectal endoscopic submucosal dissection (ESD). Best Pract Res Clin Gastroenterol. 2017;31:473-80

151. Hayashi N, Tanaka S, Hewett DG, et al. Endoscopic prediction of deep submucosal invasive carcinoma: validation of the narrow-band imaging international colorectal endocopic (NICE) classification. Gastrointest Endosc. 2013;78:625-32

152. Backes Y, Moons LM, van Bergeijk JD, et al. Endoscopic mucosal resection (EMR) versus endoscopic submucosal dissection (ESD) for resection of large distal non-pedunculated colorectal adenomas (MATILDA-trial): rationale and design of a multicenter randomized clinical trial. BMC Gastroenterol. 2016;16:56-71.

153. Thirumurthi S, Raju GS. Management of polypectomy complications Gastrointest Endosc. 2015;25:335-57.

154. Kim HS, Kim TI, Kim WH, et al. Risk factors for immediate postpolypectomy bleeding of the colon: a multicenter study. Am J Gastroenterol. 2006;101:1333-41.

155. Watabe $\mathrm{H}$, Yamaji $\mathrm{Y}$, Okamoto $\mathrm{M}$, et al. Risk assessment for delayed hemorrhagic complication of colonic polypectomy: polyp-related factors and patient-related factors. Gastrointest Endosc. 2006;64:73-8.

156. Lin D, Soetikno RM, McQuaid K et al. Risk factors for postpolypectomy bleeding in patients receiving anticoagulation or antiplatelet medications. Gastrointest Endosc. 2018;87:1106-13.

157. Fahrtash-Bahin F, Holt BA, Jayasekeran V, et al. Snare tip soft coagulation achieves effective and safe endoscopic hemostasis during wide-field endoscopic resection of large colonic lesions. Gastrointest Endosc. 2013;78:158-63.

158. Sethi A, Song LM. Adverse events related to colonic endoscopic mucosa resection and polipectomy. Gastrointest Endosc Clin N Am. 2015; 25:55-69.

159. Alcaide N, Peñas-Herrero I, Sancho-del-Val L, et al. Ovesco system for treatment of postpolypectomy bleeding after failure of conventional treatment. Rev Esp Enferm Dig. 2014;106:55-8.

160. Alsowaina KN, Ahmed MA, Alkhamesi NA, et al. Management of colonoscopic perforation: a systematic review and treatment algorithm. Surg Endosc. 2019;33:3889-98.

161. Reumkens A, Rondagh EJ, Bakker CM, et al. Post-colonoscopy complications: A systematic review, time trends, and meta-analysis of population-based studies. Am J Gastroenterol. 2016;111:1092-101.

162. Kim SY, Kim HS, Park HJ. Adverse events related to colonoscopy: Global trends and future challenges. World J Gastroenterol. 2019;25:190-204.

163. Kim ER, Chang DK. Management of complications of colorectal submucosal dissection. Clin Endosc. 2019;52:114-19.

164. Shin YJ, Kim YH, Lee KH, et al. CT findings of post-polypectomy coagulation syndrome and colonic perforation in patients who underwent colonoscopic polypectomy. Clin Radiol. 2016:71:1030-6.

165. Ito S, Hotta K, Imai K, et al. Risk factors of post-endoscopic submucosal dissection electrocoagulation syndrome for colorectal neoplasm. J Gastroenterol Hepatol. 2018;33:2001-6. 